UNIVERSIDADE DE SÃO PAULO - USP

FACULDADE DE MEDICINA DE RIBEIRÃO PRETO - FMRP

DEPARTAMENTO DE MEDICINA SOCIAL

PROGRAMA DE PÓS-GRADUAÇÃO EM SAÚDE NA COMUNIDADE

\title{
ESTUDO DO RISCO DE ÓBITO POR MEIO DA ANÁLISE DE COMORBIDADE NOS PACIENTES INTERNADOS NOS HOSPITAIS GERAIS DO DRS XIII EM 2011
}

ISABELLE CARVALHO

RIBEIRÃO PRETO 


\section{ESTUDO DO RISCO DE ÓBITO POR MEIO DA ANÁLISE DE COMORBIDADE NOS PACIENTES INTERNADOS NOS HOSPITAIS GERAIS DO DRS XIII EM 2011}

Dissertação de mestrado apresentada ao Programa de Pós-Graduação em Saúde na Comunidade, da Universidade de São Paulo, como parte dos requisitos para a obtenção do título de Mestre em Ciências - Programa de Saúde na Comunidade.

Orientador:

Prof. Dr. Juan Stuardo Yazlle Rocha

Coorientador:

Prof. Dr. Domingos Alves 


\title{
ESTUDO DO RISCO DE ÓBITO POR MEIO DA ANÁLISE DE COMORBIDADE NOS PACIENTES INTERNADOS NOS HOSPITAIS GERAIS DO DRS XIII EM 2011
}

\author{
ISABELLE CARVALHO
}

Aprovado por:

Prof. Dr. Juan Stuardo Yazlle Rocha

Faculdade de Medicina de Ribeirão Preto - Universidade de São Paulo

Prof. Dr. Milton Roberto Laprega

Faculdade de Medicina de Ribeirão Preto - Universidade de São Paulo

Profa. Dra. Cássia Maria Buchalla

Faculdade de Saúde Pública - Universidade de São Paulo 
Aos meus pais, Antônio e Rose e minha irmã Thaisa.

Obrigada pelo carinho, paciência, amor e por estarem sempre aо meu lado. 


\section{AGRADECIMENTOS}

Agradeço a Deus, pela saúde e força para superar as dificuldades e conseguir concluir mais essa etapa de minha vida.

Aos meus pais, Antônio e Rose. Pai, Mãe, muito obrigada por acreditarem em mim.

À minha irmã, Thaisa, pelo incentivo e apoio incondicional.

À minha cachorrinha, Nina, pela alegria contagiante.

Às minhas amigas Letícia Ázar e Ana Camila Magon. Obrigada por me mostrarem que mesmo longe e com as responsabilidades aumentando amigos verdadeiros caminham juntos.

À minha amiga Natália Chiari. Obrigada por compartilhar conquistas e dificuldades durante todos esses anos. Acreditamos juntas que iríamos conseguir, enfim, chegamos.

Aos meus colegas de trabalho, André, Newton e Thiago. Obrigada pela compreensão.

Agradeço a CAPES (Coordenação de Aperfeiçoamento de Pessoal de Nível Superior) pela concessão da bolsa durante um período da realização deste mestrado.

Aos meus orientadores Prof. Dr. Juan Stuardo Yazzle Rocha e Prof. Dr. Domingos Alves, pela orientação, dedicação, paciência e por me ensinarem o prazer da pesquisa, muito obrigada!

Por fim, agradeço a todos que me ajudaram e torceram por mim para a realização desse sonho.

Com carinho, Isabelle Carvalho 
“A tarefa não é tanto ver aquilo que ninguém viu, mas pensar o que ninguém ainda pensou sobre aquilo que todo mundo vê." (Arthur Schopenhauer) 


\section{RESUMO}

O trabalho apresenta o risco de óbito, baseado no estudo da comorbidade, nos pacientes internados nos hospitais gerais que oferecem assistência hospitalar na região de Ribeirão Preto adstrita ao Departamento Regional de Saúde XIII - DRS XIII. O risco de óbito foi estimado por meio do cálculo do Índice de Comorbidade de Charlson (ICC). O ICC emprega pesos de 0 a 6 a comorbidades selecionadas, ponderando o risco de morrer. Quanto maior for a pontuação do estrato do paciente, maior a chance de morrer. Para análise, foi desenvolvida uma ferramenta computacional que automatiza o cálculo do índice a fim de auxiliar os gestores na tomada de decisão. Assim, obtivemos a distribuição dos casos estudados por estrato ICC e ICCI - outra abordagem do índice ICC, quando a idade é considerada junto ao vetor de comorbidades - para o ano de 2011. Notamos que para o cálculo do ICC, a curva de risco de óbito não seguiu o previsto por Charlson, cuja mortalidade deveria aumentar à medida que a pontuação do estrato aumenta, porém, a distribuição de casos por ICCI seguiu esse conceito. Por fim, estudamos a distribuição espacial dos casos de alta gravidade que nos mostrou as cidades pólos e subpólos da região em assistência hospitalar. $\mathrm{O}$ indicador e a automatização de seu cálculo apresentou-se um bom auxílio na tomada de decisão nas questões de saúde.

Palavras chaves: Índice de Comorbidade de Charlson, Óbito, Comorbidade, Risco. 


\begin{abstract}
The work presents the risk of death, based on the study of comorbidity in hospitalized patients in general hospitals that offer hospital care in the region of Ribeirao Preto enrolled in Regional Department of Health 13th - DRS XII. The risk of death was estimated by means of the calculation of the Index of Charlson Comorbidity (ICC). The ICC employs weights from 0 to 6 the comorbidities selected, considering the risk of dying. The higher the score on the stratum of the patient, the greater the chance of dying. For analysis, a computational tool that automates the calculation of the index was developed, in order to assist managers in decision making. Thus, we obtained the distribution of cases studied by stratum ICC and ICCI another approach to the ICC index, when the age is considered along the vector of comorbidities - for the year 2011. We note that for the calculation of the ICC, the curve of risk of death did not follow the prescribed by the Charlson index, whose mortality should increase as the score of the stratum increases, however, the distribution of cases by ICCI followed this concept. The indicator and the automation of its calculation proved to be a good aid in decision-making on health issues.
\end{abstract}

Palavras chaves: Index of Charlson Comorbidity, Death, Comorbidity, Risk. 


\section{LISTA DE FIGURAS}

Figura 1- Interface inicial da ferramenta de ICC/ICCI incorporada ao portal web ORAH. ..............................29

Figura 2 - Ferramenta ICC/ICCI alocada no Portal ORAH. Exemplo de saída dos dados - tabela...................... 30

Figura 3 - Ferramenta ICC/ICCI alocada no Portal ORAH. Exemplo de saída dos dados - gráfico. ....................30

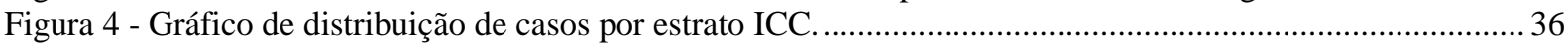

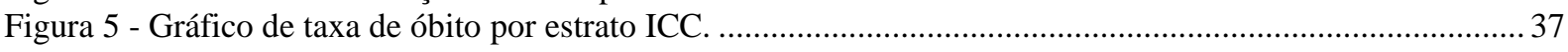

Figura 6 - Gráfico de distribuição de casos por estrato ICCI. ................................................................ 37

Figura 7 - Gráfico de taxa de óbito por estrato ICCI. .......................................................................... 38

Figura 8 - Gráfico de relação entre as taxas de óbitos com o tipo de atendimento por estrato ICC. .....................38

Figura 9 - Gráfico da relação entre as taxas de óbito com o tipo de atendimento por estrato ICCI. ....................3 39

Figura 10 - Gráfico de taxa de óbito por estrato ICCI para apresentar a gravidade dos casos: (a) Casos de baixa

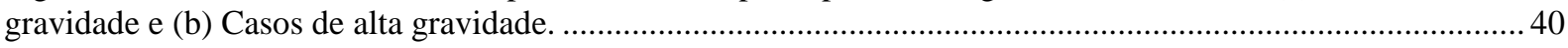

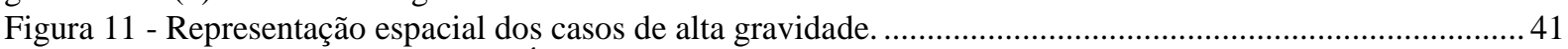

Figura 12 - Aprovação do Comitê de Ética HCFMRP . ...........................................................................48 


\section{LISTA DE TABELAS}

Tabela 1 - Condições clínicas que compõem o ICC e os respectivos pesos incorporados a elas.

Tabela 2 - Pontuação referente à idade da paciente a ser considerada junto as comorbidades no ICCI...............21

Tabela 3 - Número de internações de acordo com a quantidade de diagnósticos anotados............................... 31

Tabela 4 - As cinco comorbidades mais frequentes e o diagnóstico principal relacionado................................ 31

Tabela 5 - Número de internações de acordo com o tipo de atendimento (SUS e não SUS) e a quantidade de

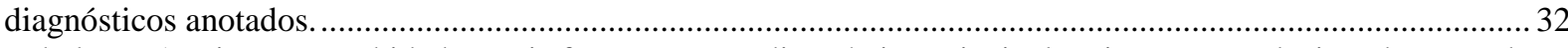

Tabela 6 - As cinco comorbidades mais frequentes e o diagnóstico principal mais comum relacionado segundo atendimento SUS.

Tabela 7 - As cinco comorbidades mais frequentes e o diagnóstico principal mais comum relacionado segundo atendimento não SUS

Tabela 8 - Número de internações de acordo com a faixa etária do paciente e a quantidade de diagnósticos anotados.

Tabela 9 - As três comorbidades mais frequentes e o diagnóstico mais comum relacionado segundo faixa etária.

Tabela 10 - Frequência das condições clínicas estudadas por Charlson. 


\section{SUMÁRIO}

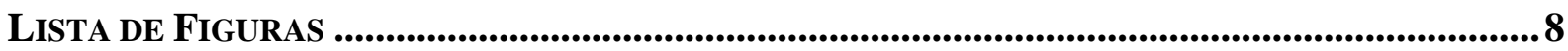

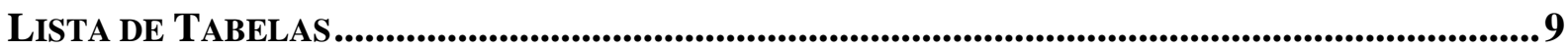

CAPÍTULO 1 ........................................................................................................................................................ 11

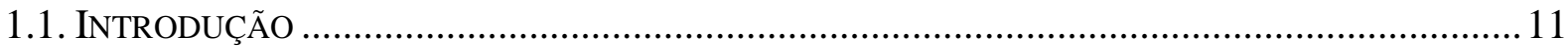

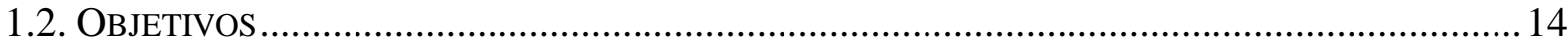

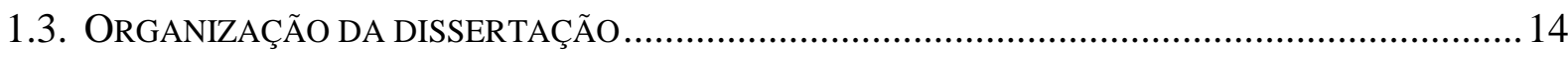

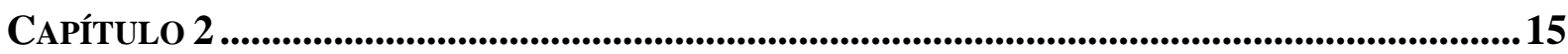

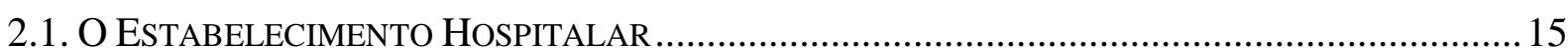

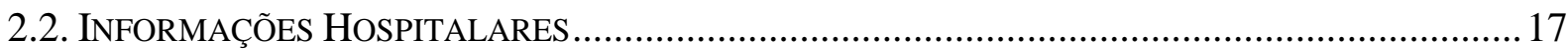

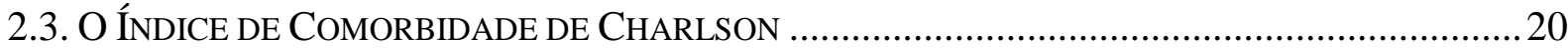

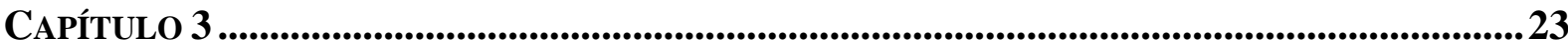

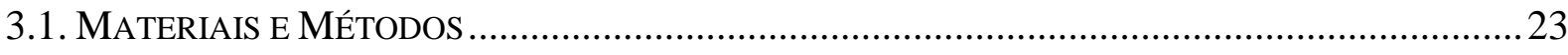

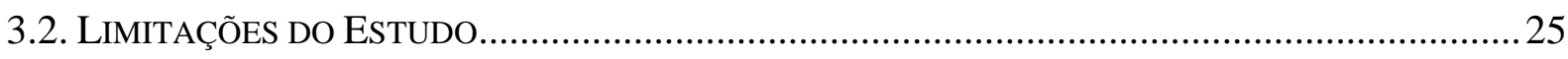

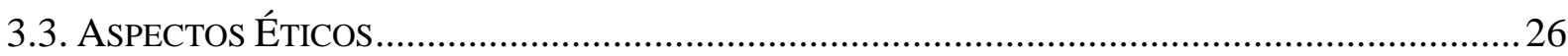

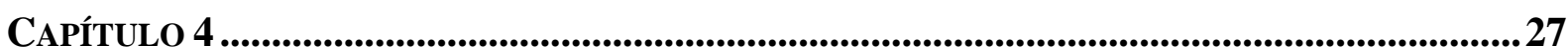

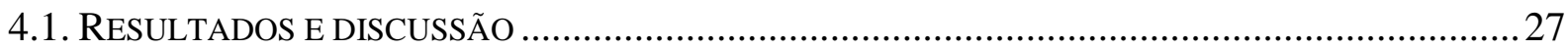

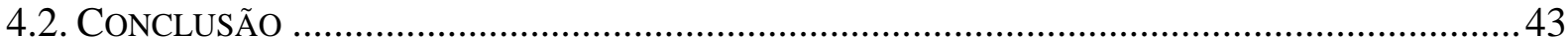

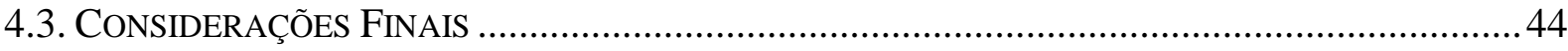

REFERÊNCIAS ..................................................................................................................................................... 45

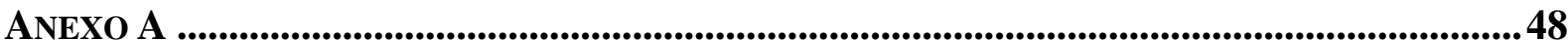




\section{Capítulo 1}

\subsection{INTRODUCÃ̃O}

Indicadores hospitalares são instrumentos utilizados para avaliar o desempenho hospitalar, envolvendo sua organização, recursos e metodologia de trabalho. Os dados coletados nas diversas áreas do hospital, quando relacionados entre si, transformam-se em instrumentos de gestão úteis para a avaliação da assistência prestada, quantidade e tipo de recursos envolvidos, controle dos custos gerados na produção dos serviços e grau de resolutividade dos mesmos (CQH, 1992). Uma ressalva a ser considerada é a grande dificuldade existente em definir e interpretar "gold standards" para a performance de hospitais.

Apesar da longa tradição dos indicadores de saúde e hospitalares, poucas instituições os utilizam de forma habitual para tomada de decisão (GONÇALVES, 2006). Porém, a utilização de indicadores com metodologia e critérios científicos, propicia aos hospitais um ganho de qualidade e eficiência em suas ações (GONÇALVES, 2006), permitindo uma série de avanços no Modelo de Gestão, através do "Benchmarking", com outras instituições, acompanhamento da evolução de seus indicadores, identificação de pontos fracos e fortes da mesma. O ganho com a utilização de indicadores no processo de gestão pode ser ainda mais eficaz, caso o acompanhamento dos mesmos seja realizado de forma constante, não somente após o fechamento periódico, mas sim no dia a dia da gestão, com avaliações diárias. Com esse intuito, algumas instituições brasileiras estão aderindo a propostas de avaliação da qualidade hospitalar de âmbito nacional como o CQH (Compromisso com a Qualidade Hospitalar) (CQH, 2006), e em outros casos as novas políticas de saúde estão obrigando as instituições a disponibilizarem periodicamente vários indicadores, com base em um Plano de Metas, onde o desempenho dos mesmos pode influenciar em suas remunerações.

Em âmbito internacional, temos a agência norte americana "Agency for Healthcare Research and Quality" (AHRQ), que preconiza alguns indicadores de qualidade hospitalar bem definidos e estudados. Os "Inpatient Quality Indicator" são um conjunto de medidas que fornecem uma perspectiva sobre a qualidade dos cuidados dentro dos hospitais, utilizando os dados administrativos dos hospitais. Estes indicadores incluem mortalidade hospitalar por algumas condições clínicas e procedimentos cirúrgicos, taxas de utilização e volume de certos procedimentos. 
A mortalidade hospitalar é um indicador tradicional de desempenho hospitalar e, provavelmente, o será por muito tempo. Ela expressa uma dimensão crucial da qualidade do cuidado prestado: o resultado final. Todavia, diferenças entre hospitais podem ser derivadas da prática médica, do perfil dos casos tratados ou da interação entre esses elementos (IUCIF JR; ROCHA, 2004). Assim, na realização de estudos comparativos, pacientes com características de saúde diferenciadas, que implicam diferentes tratamentos, não podem ser analisados como similares e um ajuste adequado na caracterização de tais pacientes é muito importante (BLUMBERG, 1986). Os fatores do risco de morte dos pacientes (gravidade) são as variáveis de ajuste das taxas de mortalidade hospitalar na realização desses tipos de estudos e em conjunto, a comparação do tempo de permanência, dos custos ou da mortalidade implica determinar em que extensão as diferenças observadas podem ser atribuídas ao tipo de caso admitido ou às diferenças no tratamento (MARTINS; TRAVASSOS; NORONHA, 2001).

Particularmente, a intensidade (número e gravidade) das doenças coexistentes constitui importante preditor de complicações e de resultados desfavoráveis. Portanto, a mensuração da gravidade representa um importante desafio metodológico. Diversos métodos para medir a gravidade dos casos foram desenvolvidos (THOMAS, 2004).

Nesse sentido, o índice de comorbidade de Charlson (ICC) é uma classificação de gravidade baseado em comorbidades. Tipicamente, os métodos de ajuste de risco que se baseiam na presença de comorbidade podem ser aplicados quando se dispõe de dados de bases administrativas, pois, apesar de não conterem outras informações clínicas, sempre fornecem informação diagnóstica. Contudo, sua utilidade depende da completude e precisão dessas informações. O ICC emprega condições clínicas selecionadas, registradas como diagnóstico secundário - comorbidades - no cálculo do risco de morrer, ou seja, o índice calcula a carga de morbidade do paciente, independentemente do diagnóstico principal. Diante disso, teremos de acordo com os pesos preconizados por Charlson (CHARLSON et al., 1987) um peso total que identifica o paciente em relação a sua gravidade perante o atendimento que deve ser prestado ao hospital.

Temos que um conjunto de informações relevantes pode descrever melhor um determinado cenário estudado e a partir disso podemos considerar que acoplando as informações do ICC a outras informações hospitalares, como por exemplo, a natureza do hospital (SUS ou não-SUS), podemos descrever a distribuição do risco de óbitos dos pacientes, levantar hipóteses sobre o atendimento oferecido e auxiliar na tomada de decisão dos gestores. 
Portanto, o trabalho pretende, preservando as características de uma pesquisa acadêmica, utilizar e aprimorar tecnologias, no caso, a manipulação do índice de comorbidade de Charlson para a análise de dados hospitalares provendo a predição de óbito hospitalar. $\mathrm{O}$ produto final esperado consiste na organização de acesso às informações, uma melhor gestão e manuseio dos dados hospitalares. Teremos então, além da análise do cenário sobre a gravidade e cuidados dos pacientes da região, uma ferramenta de auxílio à gestão com o intuito de melhorar as decisões tomadas e consequentemente os serviços prestados. 


\subsection{OBJETIVOS}

\subsubsection{GERAL}

O presente trabalho tem como objetivo estudar a distribuição do risco de óbito dos pacientes internados nos hospitais gerais da região de Ribeirão Preto adscrita a DRS XIII no ano de 2011 segundo a comorbidade.

\subsubsection{ESPECÍFICOS}

- Utilizar e aprimorar tecnologias que proporcionem a automatização do cálculo do ICC e consequentemente a manipulação de dados hospitalares;

- Caracterizar as comorbidades referentes as internações do estudo;

- Apresentar, por meio do ICC e ICCI, a distribuição do risco de óbito dos hospitais gerais pertencentes ao DRS XIII no ano de 2011.

\subsection{ORGANIZAÇÃO DA DISSERTAÇÃO}

A dissertação é composta por quatro capítulos. O Capítulo 2 apresenta os conceitos envolvidos no trabalho. O Capítulo 3 apresenta os materiais e métodos que foram utilizados no decorrer da pesquisa, os aspectos éticos e as limitações do estudo. O Capítulo 4 apresenta os resultados e discussão do tema, a conclusão e as considerações finais do autor. Por fim, temos as referências bibliográficas e os anexos. 


\section{Capítulo 2}

\subsection{O ESTABELECIMENTO HOSPITALAR}

O hospital mais antigo do país é a Santa Casa de Santos, fundado por Braz Cubas, em 1543 (MINISTÉRIO DA SAÚDE, 1990). A palavra hospital é de origem latina, vem de "hospes", que significa hóspede, pois na antiguidade era o lugar onde se hospedavam, além de enfermos, viajantes e peregrinos (MINISTÉRIO DA SAÚDE, 1965). Atualmente, temos hospital como:

[...] parte integrante de uma organização médica e social, cuja função básica consiste em proporcionar à população assistência médica integral, curativa e preventiva, sob quaisquer regimes de atendimento, inclusive o domiciliar, constituindo-se também em centro de educação, capacitação de recursos humanos e de pesquisas em saúde, bem como de encaminhamento de pacientes, cabendo-lhe supervisionar e orientar os estabelecimentos de saúde a ele vinculados tecnicamente [...] (MINISTÉRIO DA SAÚDE, 1977).

A fim de compor conhecimento sobre os hospitais e facilitar a adoção de políticas e planejamento de ações em saúde eles podem ser classificados quanto à natureza assistencial, quanto à capacidade, quanto ao tipo de atendimento e de diversas outras maneiras de acordo com características semelhantes. (MINISTÉRIO DA SAÚDE, 2002a).

Quanto à natureza assistencial, os hospitais podem ser gerais ou especializados. Os gerais são destinados a atender pacientes portadores de doenças das várias especialidades médicas e ter a sua ação limitada a um grupo etário (hospital infantil), a determinada camada da população (hospital militar, hospital previdenciário) ou a finalidade específica (hospital de ensino), enquanto os hospitais especializados são destinados, predominantemente, a atender pacientes necessitados da assistência a uma determinada especialidade médica. (MINISTÉRIO DA SAÚDE, 1977).

Quanto à capacidade, os hospitais podem ser de pequeno, médio ou grande porte. Hospitais de pequeno porte possuem capacidade de operação para até 50 leitos, os de médio porte atingem de 50 a 150 leitos e os de grande porte de 150 a 500 leitos. Acima de 500 leitos o hospital é considerado de capacidade extra. Os termos pequeno, médio, grande e extra, se referem unicamente ao número de leitos, não tendo qualquer relação com a qualidade e/ou complexidade da assistência prestada nesses estabelecimentos (MINISTÉRIO DA SAÚDE, 1977). 
Quanto ao tipo de atendimento, o cuidado é financiado pelo setor público ou privado. O setor público compreende o Sistema Único de Saúde (SUS) que tem como base os princípios da universalidade, integralidade, equidade, descentralização e participação social. O SUS foi instituído constitucionalmente em 1988 e regulamentado por meio das Leis Orgânicas de Saúde (Lei 8080 e Lei 8142) (MINISTÉRIO DA SAÚDE, 1990). O setor privado age no sistema suplementar de saúde e agrega instituições como empresas, cooperativas, de autogestão que operam com planos de saúde e com os prestadores de serviços privados da área (MINISTÉRIO DA SAÚDE, 2000; SANTOS; UGÁ; PORTO, 2008).

Cabe entendermos, no entanto, onde um hospital se encaixa no contexto de atenção ao paciente. Há três níveis de atenção - primário, secundário e terciário - que se estruturam como arranjos produtivos segundo o tipo de atendimento prestado e os recursos oferecidos.

A atenção primária abrange uma pequena área territorial e conta com os agentes comunitários de saúde e com as unidades básicas de saúde para oferecer atendimentos de baixa complexidade. A atenção secundária reúne os serviços especializados, de apoio diagnóstico e terapêutico, e esses serviços podem estar centralizados em hospitais gerais, com grau intermediário de inovação tecnológica, ou em centros de especialidades médicas (AMEs). No nível secundário a área de abrangência é representada por um distrito sanitário que é uma área geográfica que comporta uma população com características sociais e demográficas e que provê recursos de saúde capazes de atender as necessidades da mesma (ALMEIDA; CASTRO; VIEIRA, 1988). Por último, a atenção terciária que tem a maior área de abrangência, sendo um município ou até vários deles e é composta pelos hospitais gerais de grande incorporação tecnológica, com equipamentos de última geração, proporcionando atendimentos de alta complexidade (MENDES, 2010). Com isso, temos os hospitais assistindo à população nos níveis secundários e terciários de atenção à saúde. 


\subsection{INFORMACÕES HOSPITALARES}

Os serviços prestados pelos hospitais geram uma grande quantidade de dados a cada minuto. Esses dados são registrados e guardados em documentos e pastas que propiciam a organização do setor hospitalar, representam características do cenário atual e referenciam o paciente, o profissional e as atividades envolvidas.

A Autorização de Internação Hospitalar (AIH) é um desses documentos pertencentes a rotina hospitalar. Ela deve ser preenchida pelos profissionais vinculados aos estabelecimentos de saúde públicos e privados, engloba as informações sobre a identificação do paciente e dos serviços prestados a ele durante suas internações e é preferencialmente baseada no laudo médico. O objetivo desse documento é o reembolso dos serviços hospitalares prestados ao paciente, por isso, alguns estudos visam à confiabilidade dos dados e levantam hipóteses de que os responsáveis pelo preenchimento possam estar dando maior importância aos procedimentos e diagnósticos que permitem maior remuneração (MATHIAS; SOBOLL, 1998; VERAS; MARTINS, 1994).

Um ponto importante a ser levantado é que para a anotação das morbidades relacionadas aos pacientes, os documentos envolvidos utilizam-se de uma padronização segundo a Classificação Internacional de Doenças (CID) - uma categorização de causas de morte a fim de permitir comparabilidade internacional (LAURENTI, 1991).

Outro documento presente na rotina hospitalar é a Folha de Alta Hospitalar ou Resumo da Internação que descreve alguns aspectos do processo assistencial durante a hospitalização. Ela contém as informações mais importantes do prontuário que se referem à identificação do hospital, enfermaria e características do paciente (YAZLLE ROCHA; SIMÕES; GUEDES, 1997). O preenchimento é feito no egresso do paciente por um responsável pela função que deve ser preferencialmente o médico assistente do caso (ROCHA, 1975).

O Centro de Processamento de Dados Hospitalares (CPDH) da Faculdade de Medicina de Ribeirão Preto (FMRP) é um exemplo de sistema de informação para facilitar o manuseio das folhas de alta hospitalares. O CPDH surgiu em 1969 com o objetivo de coletar, processar e garantir a qualidade das informações de internação hospitalar por meio de protocolos internos de verificação e correção de inconsistências. Os dados fornecidos pelo Centro abrangem 34 hospitais localizados na região de Ribeirão Preto - adstrita ao Departamento Regional de Saúde XIII (DRS XIII) - e processa anualmente cerca de 170 mil internações hospitalares, das quais $60 \%$ são financiadas pela assistência pública provida pelo SUS. 
Em 2009, com base nos dados que envolvem o CPDH, surgiu o Observatório Regional de Atenção Hospitalar - ORAH, uma iniciativa da Secretaria de Estado da Saúde de São Paulo por meio do DRS XIII em parceria com a FMRP, que tem como objetivo principal garantir que as informações em saúde, sobretudo sobre assistência hospitalar, ganhem em qualidade com desdobramentos para a assistência, gestão e pesquisa em saúde. O ORAH, um portal web de conteúdo, se tornou capaz de auxiliar na tomada de decisão de médicos e gestores de saúde por meio de ferramentas computacionais desenvolvidas especificamente para subsidiar o arranjo das informações hospitalares (CARVALHO et al., 2013).

Diante disso, as informações geradas durante o cuidado do paciente devem subsidiar a construção do conhecimento a fim de auxiliar na avaliação da assistência hospitalar. A avaliação da assistência em saúde se iniciou no Brasil por meio do Censo Hospitalar que serviu de inspiração por incorporar conceitos de regionalização e hierarquização de serviços no processo de monitoramento da atenção prestada ao paciente (FELDMAN; GATTO; CUNHA, 2005). O processo de apoio no monitoramento dos serviços em saúde atualmente tem o auxílio dos sistemas de informações a saúde para a manipulação dos dados e, consequentemente, na construção de indicadores capazes de subsidiar a tomada de decisão e avaliar a assistência hospitalar de maneira mais confiável e eficaz.

Um indicador de saúde serve para medir aspectos não sujeitos à observação direta, isto é, deve revelar a situação de saúde de um indivíduo ou de uma população de forma a quantificar processos e produtos assistenciais. O objetivo é controlar e melhorar a qualidade e o desempenho da assistência (AMAZONAS, 2012; TREVISO; BRANDÃO; SAITOVITCH, 2009).

Primeiramente deve-se delimitar o problema a ser observado e após isso escolher o indicador apropriado que deve ser capaz de discriminar o evento de interesse de outros, de detectar mudanças ocorridas ao longo do tempo e de obter resultados semelhantes quando a medição é repetida (AMAZONAS, 2012).

Eles podem, por exemplo, embasar a análise crítica dos resultados e do processo de tomada de decisão; facilitar o planejamento e o controle do desempenho; identificar características e atributos que diferenciam um serviço daquele oferecido pela concorrência; conhecer a percepção do cliente externo; viabilizar a análise comparativa do desempenho da organização; diagnosticar os pontos fortes e fracos do serviço ou avaliar comparativamente diferentes organizações (TREVISO; BRANDÃO; SAITOVITCH, 2009).

Por exemplo, os coeficientes de mortalidade e de letalidade são tipos de indicadores que auxiliam à tomada de decisão e ambas representam informações que os hospitais, os 
profissionais e os financiadores devem usar para melhor entender o processo de atendimento ao paciente (TRAVASSOS; NORONHA; MARTINS, 1999).

A taxa ou coeficiente de mortalidade hospitalar é a relação percentual entre o número de óbitos ocorridos em pacientes internados e o número de pacientes que tiveram saída do hospital, em determinado período e tem como objetivo medir a proporção dos pacientes que morreram durante a internação hospitalar (MINISTÉRIO DA SAÚDE, 2002b). A letalidade relaciona o número de óbitos por determinada causa e o número de pessoas que foram acometidas por tal doença. Essa relação entre o percentual de pessoas que morreram por tal doença nos dá a ideia de gravidade e pode informar sobre a qualidade da assistência médica oferecida à população (PEREIRA, 2007).

Isto posto, o trabalho descrito visa o estudo do risco dos óbitos dos pacientes no contexto hospitalar da região de Ribeirão Preto. A exploração do assistência hospitalar foi feita com o auxílio de um indicador de saúde baseado nas informações sobre as comorbidades do paciente anotadas durante o egresso do paciente na Folha de Alta Hospitalar. 


\subsection{O ÍNDICE DE COMORBIDADE DE CHARLSON}

Comorbidades são definidas como condições médicas coexistentes que são distintas da condição primária sob investigação e influenciam no cuidado do paciente durante a detecção, terapia e resultado (FARLEY; HARLEY; DEVINE, 2006). Elas podem compartilhar com a morbidade o mesmo fator de risco, ser causa ou consequência de uma doença e também interferir no prognóstico e no seguimento de um ensaio clínico randomizado (DE GROOT et al., 2003).

A exploração das comorbidades consegue, entre outras coisas: (i) corrigir confusão em estudos clínicos; (ii) identificar modificações no efeito apresentado; (iii) estudar predição de morte, prognóstico e/ou história natural e (iv) permitir aplicação de métodos estatísticos para levantar hipóteses (DE GROOT et al., 2003).

Nesse sentido, QUAIL et al. (2011) afirmam que para as pesquisas relacionadas a eventos e serviços relativos à saúde é essencial estudarmos o ajuste do risco para comorbidades a fim de obtermos estimativas imparciais. Os autores analisaram cinco medidas de ajuste de risco baseadas em comorbidades e concluíram que o melhor indicador para medir comorbidade depende do resultade de interesse do pesquisador e não da população de estudo. QUAIL et al. (2011) apresentaram também que em três populações de estudo - duas coortes de estudo referentes a doenças crônicas (diabetes e osteoporose) e a população geral - o índice Elixhauser seguido pelo Índice de Comorbidade de Charlson (ICC) tiveram o melhor desempenho preditivo de mortalidade. O índice Elixhauser explora 30 comorbidades e foi desenvolvido para prever duração da estadia, custo e óbito hospitalar (CHU; NG; WU, 2010; DE GROOT et al., 2003). O ICC será descrito em detalhes, pois é o indicador escolhido como base desse estudo.

O Índice de Comorbidade de Charlson (ICC) foi definido em 1987 por Mary E. Charlson e colaboradores para classificar a gravidade dos pacientes. Esse método pode ser aplicado em bases de dados administrativos e emprega condições clínicas registradas como diagnóstico secundário - comorbidades - no cálculo do risco de óbito. Portanto, o índice é um preditor de óbito que calcula a carga de morbidade do paciente, independentemente do diagnóstico principal.

Para compor o ICC, foram definidas 17 condições clínicas influenciáveis na chance do paciente vir a falecer e para cada uma delas um peso variando de zero a seis (IUCIF JR; ROCHA, 2004). As condições clínicas e os pesos que compõem o índice foram selecionados por apresentarem um risco relativo, derivado da análise de sobrevida, maior que 1,2, no 
conjunto de 30 condições clínicas originalmente estudadas em um estudo coorte com 604 casos de câncer de mama do New York Hospital (Nova York, Estados Unidos) (CHARLSON et al., 1987; MARTINS; BLAIS; MIRANDA, 2008). A Tabela 1 apresenta as 17 condições que compõem o ICC e os respectivos pesos incorporados a elas.

Tabela 1 - Condições clínicas que compõem o ICC e os respectivos pesos incorporados a elas.

\begin{tabular}{c|c}
\hline Pontuação & Condições Clínicas \\
\hline 1 & Infarto Agudo do Miocárdio \\
\hline 1 & Insuficiência Cardíaca Congestiva \\
\hline 1 & Doença Vascular-Periférica \\
\hline 1 & Acidente Vascular Cerebral (AVC) \\
\hline 1 & Demência \\
\hline 1 & Doença Pulmonar \\
\hline 1 & Doença do tecido conjuntivo \\
\hline 1 & Doença hepática \\
\hline 1 & Diabetes sem complicações \\
\hline 1 & Diabetes com complicações \\
\hline 2 & Hemiplegia ou paraplegia \\
\hline 2 & Doença renal severa ou moderada \\
\hline 2 & Câncer \\
\hline 2 & Câncer em metástase \\
\hline 3 & Doença do fígado moderada à grave \\
\hline 3 & AIDS \\
\hline 6 & \\
\hline
\end{tabular}

Durante o estudo, Charlson et at. observou que além das comorbidades, a idade também era um agravante na predição de óbito, principalmente em estudos longitudinais de maior duração. Com isso, em 1994, o ICC regular foi adaptado e criou-se o ICC ajustado por idade (ICCI) onde a idade é considerada junto ao vetor de comorbidades e a partir de 50 anos, a cada década da vida, é adicionado um peso como apresentado na Tabela 2 (CHARLSON et al., 1994; IUCIF JR; ROCHA, 2004; THOMAS, 2004).

Tabela 2 - Pontuação referente à idade da paciente a ser considerada junto as comorbidades no ICCI.

\begin{tabular}{c|c}
\hline Pontuação & Grupo etário \\
\hline 0 & $0-49$ anos \\
\hline 1 & $50-59$ anos \\
\hline 2 & $60-69$ anos \\
\hline 3 & $70-79$ anos \\
\hline 4 & $80-89$ anos \\
\hline 5 & Mais de 90 anos \\
\hline
\end{tabular}


Diante disso, o escore de gravidade do paciente é o resultado da soma dos pesos dos componentes do índice e a interpretação visa que a pontuação que o paciente obteve no ICC ou ICCI é a quantidade de chances de vir a falecer comparado a outro paciente sem essas comorbidades (CHARLSON et al., 1987). Ou seja, a pontuação ICC ou ICCI do paciente é relacionada a pontuação definida por Charlson et. al. e o paciente se alocará em um estrato com pontuação variante de 0 a maior que 6 onde quanto maior o estrato de alocação, maior é sua chance de vir a óbito.

No Brasil, o uso de medidas de risco para o ajuste de indicadores de resultado dos cuidados de saúde é pouco frequente, sendo também recente o emprego de base de dados administrativos para avaliar a qualidade dos serviços de saúde (IUCIF JR; ROCHA, 2004; MARTINS; BLAIS; LEITE, 2004; MARTINS; BLAIS; MIRANDA, 2008; TRAVASSOS; NORONHA; MARTINS, 1999).

No que diz respeito ao ICC, os trabalhos visam avaliar a confiabilidade dessa metodologia e se ela reflete a mortalidade hospitalar através de critérios relevantes. IUCIF JR; ROCHA (2004) estudaram a desigualdade na mortalidade hospitalar pelo índice de comorbidade de Charlson e revelaram que o risco de morte é mais do que o dobro para os pacientes SUS comparados com os do não-SUS, quando esse analisava a mortalidade por meio dos critérios de Charlson, entre os pacientes idosos atendidos nesses dois eixos de atenção hospitalar. MARTINS; BLAIS; LEITE (2004) usou o ICC para avaliar a mortalidade hospitalar e o tempo de permanência de pacientes cardiovasculares e respiratórios, sendo que o critério principal de análise foi a influência das comorbidades presentes nas internações. Em ambos os casos, os estudos foram realizados utilizando a base de dados de internações coletados pelo Centro de Processamento de Dados (CPDH) do Hospital das Clínicas da Faculdade de Medicina de Ribeirão Preto (HCFMRP - USP).

TRAVASSOS; NORONHA; MARTINS (1999) fizeram uma revisão das condições e pesos propostos por Charlson, propondo adaptações, e destacando a importância da utilização de um maior número de campos para registro de comorbidades, endossado por um estudo mais específico ao se utilizar da base de dados do Sistema de Informações Hospitalares do Sistema Único de Saúde (SIH-SUS) (MARTINS; TRAVASSOS; NORONHA, 2001).

Como já mencionado, o ICC é considerado pela literatura um importante preditor de mortalidade e por isso, a partir dele obtivemos as distribuições de risco de óbito das internações pertencentes a esse estudo. 


\section{Capítulo 3}

\subsection{MATERIAIS E MÉTODOS}

\subsubsection{FonTE DE DAdOS}

A base de dados utilizada provém do sistema de informação do Observatório Regional de Assistência Hospitalar - Portal ORAH, alimentada pelo Centro de Processamentos de Dados do Hospitalares (CPDH), do Hospital das Clínicas da Faculdade de Medicina de Ribeirão Preto (HCFMRP - USP). Particularmente, as informações utilizadas foram sobre as internações nos hospitais gerais pertencentes a região de Ribeirão Preto, adstrita ao Departamento Regional de Saúde XIII (DRS XIII), no ano de 2011. Essa base de dados é baseada na Folha de Alta Hospitalar e contém informações sobre o perfil demográfico dos pacientes; os diagnósticos principal e secundários; os procedimentos cirúrgicos, terapêuticos e diagnósticos; a especialidade médica do caso tratado; as datas de entrada e saída; o tipo de alta e a fonte de pagamento. As informações, embora estejam disponíveis para cada paciente, são anônimas, de modo que é impossível identificar os pacientes e elas abrangem uma cobertura universal de todas as hospitalizações, seja esta financiada ou não pelo Estado. Uma característica importante da base de dados é que ela permite o registro da informação de até 4 diagnósticos secundários. Vale ressaltar que as informações do $\mathrm{CPDH}$ são conferidas, codificadas e processadas eletronicamente passando por um programa de consistência que verifica 33 tipos de incoerências, contradições e inconsistências (SIMÖES et al., 1991) e que somente após consolidadas elas são inseridas no banco de dados do Portal.

\subsubsection{O ÍNdice de Comorbidade de Charlson (ICC) E O ÍNDICE DE Comorbidade DE ChaRlson AJUSTAdo PARA IdAde (ICCI)}

No presente estudo, para cada internação, foi aplicada a ponderação conforme o número e intensidade dos diagnósticos secundários, seguindo a metodologia preconizada por Charlson para as comorbidades e para idade (BLUMBERG, 1986; CHARLSON et al., 1994, 1987).

Charlson originalmente examinou 17 condições clínicas, codificadas de acordo com a CID versão 9 (CHARLSON et al., 1987) e para os cálculos serem aplicáveis a base de dados 
do estudo foi necessário representar essas condições por meio da CID versão 10, o padrão de codificação para diagnósticos utilizado na base de dados do Portal ORAH.

Assim, uma ferramenta computacional que permite o cálculo do ICC e do ICCI foi desenvolvida. Ela foi construída em linguagem Hypertext Preprocessor (PHPC, 2013) e recursos da tecnologia HyperText Markup Language (HTML, 2013) e Cascading Style Sheets (CSS, 2013), devido à adequação para desenvolvimento web e a fim de seguir os padrões previamente implantados no Portal ORAH. Além de permitir a automação do cálculo do índice, a ferramenta permite também a exploração dos dados por meio de filtros de consultas específicos, como: sexo, causa de internação (diagnóstico principal) e tipo de atendimento (SUS e não SUS).

\subsubsection{A Distribuição do Risco de ÓbITO BASEAdo NA PONDERAÇÃO DAS COMORBIDADES}

Com o auxílio da ferramenta, o trabalho apresenta a distribuição do risco de óbito baseado na ponderação das comorbidades dos pacientes durante suas internações nos hospitais gerais pertencentes ao DRS XIII. Para isso, preliminarmente fizemos um estudo descritivo das comorbidades presentes no banco de dados.

Então, aplicamos a ferramenta para o cálculo do ICC e ICCI nos dados dos hospitais participantes do estudo e obtivemos as distribuições de casos de acordo com cada estrato ICC e ICCI. Vale ressaltar que quanto maior o estrato (maior pontuação ICC ou ICCI), maior a gravidade do caso em questão e maior a chance de óbito. Para estudarmos a gravidade, em conjunto com as distribuições dos casos, calculamos as taxas de óbitos nos estratos ICC e ICCI, com o propósito de observarmos se o risco de óbito se ajustava com a real situação na distribuição de mortalidade. A partir disso, foram considerados como sendo mais graves os casos que estavam alocados nos estratos com pontuação acima do ponto médio da distribuição da taxa de óbito.

No final, obtivemos as distribuições dos casos por tipo de atendimento (SUS e não SUS) e por hospital para estudarmos a distribuição espacial dos casos mais graves. 


\subsection{LIMITACÕES DO ESTUDO}

O trabalho utilizou dados secundários, ou seja, nós não consultamos o prontuário do paciente. Essa abordagem carrega, então, os vieses que circundam o processo de coleta, codificação e digitação do CPDH para a construção da base de dados. Atualmente, um estudo está em andamento para medir a qualidade de cada etapa desse processo e consequentemente dar maior confiabilidade aos dados (VINCI, 2012).

No que se refere à ferramenta construída, ela pode ser aplicada a qualquer base de dados de hospitalizações que contenha as informações básicas para o cálculo do indicador. Porém, os dados envolvidos como matéria prima devem seguir a mesma padronização, isto é, os diagnósticos e principalmente as comorbidades relacionadas ao paciente e/ou internação devem estar codificadas de acordo com a Classificação Internacional de Doenças, versão 10.

Por fim, um ponto a ser levantado é sobre as anotações de comorbidades em nossos serviços de saúde. Infelizmente, essa prática ainda não está bem incorporada aqui no Brasil, pois na Autorização de Internação Hospitalar - documento padrão para a identificação de uma internação - é reservado apenas um campo para a anotação de comorbidades, o que dificulta a exploração de estudos nessa área (MARTINS, 2010). 


\subsection{Aspectos Éticos}

A pesquisa segue as exigências éticas prescritas pela resolução CNS 196/96 e suas complementares. Os pesquisadores estão cientes e cumpriram os conteúdos desta portaria. Vale ressaltar que a pesquisa não envolveu experimentação em seres humanos, nem tem foco em grupos especiais.

Em anexo segue a carta de aprovação do Comitê de Ética da Unidade de Pesquisa Clínica do Hospital das Clínicas de Ribeirão Preto para a realização da pesquisa (Anexo A). 


\section{Capítulo 4}

\subsection{RESULTADOS E DISCUSSÃO}

Essa seção apresenta os resultados obtidos e está disposta em 5 subseções: (4.1.1.) Universo de Estudo que descreve o cenário do trabalho; (4.1.2.) Ferramenta Computacional ICC/ICCI que apresenta a automatização do cálculo do Índice de Comorbidade de Charlson; (4.1.3.) Caracterização das Comorbidades que estuda o comportamento das comorbidades no conjunto de dados; (4.1.4.) Caracterização da Distribuição de Risco de Óbito com Base no ICC/ICCI que explora os dados de acordo com os índices do estudo e, (4.1.5.) Trabalhos Apresentados em Eventos que apresenta os trabalhos resultantes dessa pesquisa expostos em eventos.

\subsubsection{UNIVERSO DO ESTUDO}

Como dito anteriormente, os dados foram provenientes da Folha de Alta Hospitalar que permite a anotação de até quatro diagnósticos secundários, um dado essencial para a pesquisa em questão baseada na ponderação da comorbidade. É importante ressaltar que os dados representam uma alta hospitalar e não um paciente e por isso iremos caracterizar o caso do paciente durante a internação.

O trabalho é um estudo do tipo descritivo e envolveu as internações disponíveis no Portal ORAH em 08/01/2014 dos hospitais gerais que tinham os dados completos no ano de 2011. Os hospitais gerais foram selecionados porque o estudo é baseado na caracterização de morbidades e comorbidades e as internações nesses estabelecimentos abrangem portadores de doenças de diversas especialidades médicas apresentando o cenário da morbidade hospitalar na região de Ribeirão Preto em 2011. São 28 hospitais gerais da região parceiros do CPDH, porém oito não apresentavam dados completos em 2011 e 1 não apresentava óbitos nesse período, assim, 19 hospitais gerais que abrangem 12 cidades pertencentes ao Departamento Regional de Saúde XIII foram explorados pelo estudo.

\subsubsection{A FERRAMENTA COMPUTACIONAL ICC/ICCI}

A primeira versão da ferramenta computacional que provia o cálculo do Índice de Comorbidade de Charlson foi desenvolvida durante o Trabalho de Conclusão de Curso em Informática Biomédica do aluno Wagner Yoshikazu Hitomi. Ela foi construída em linguagem 
Java (ORACLE, 2013) e apresentava como resultado, depois de realizada uma consulta, uma tabela contendo as informações gerais de cada alta hospitalar e seu respectivo valor de ICC e ICCI (HITOMI et al., 2012).

Para a incorporação da ferramenta no Portal ORAH, identificou-se a necessidade de realizar melhorias na primeira versão, tanto no que se refere às questões técnicas de programação, quanto no que diz respeito à adequação de alguns conceitos. Vale ressaltar, aqui, que a autora iniciou algumas dessas melhorias em conjunto a realização do trabalho de conclusão de curso supracitado, sendo que uma delas consistiu na conversão do padrão de codificação CID 9 - utilizado originalmente para codificar as 17 condições clínicas de Charlson - para CID 10 - padrão utilizado nos dados desse estudo (QUAN et al., 2005).

Ainda no que tange ao aprimoramento da ferramenta para a incorporação desta no Portal ORAH, sua nova versão apresenta interfaces gráficas de interação com o usuário - as quais não estavam presentes na versão anterior - que são fundamentais para uma melhor usabilidade da ferramenta. Além disso, ela foi recodificada na linguagem PHP para se conformar aos padrões de desenvolvimento já existentes no Portal. No que se refere às novas funcionalidades da ferramenta, a nova versão provê filtros de consulta que permitem permutar os valores das variáveis de entrada dinamicamente e em tempo real e expõe os resultados de uma consulta em forma de tabelas e gráficos, garantindo, assim, maior precisão e detalhamento dos resultados.

A nova versão da ferramenta para o cálculo do Índice de Comorbidade de Charlson está disponível em http://ciis.fmrp.usp.br/orah. A Figura 1 apresenta a interface inicial da ferramenta de cálculo do Índice de Comorbidade de Charlson e Índice de Comorbidade de Charlson corrigido por Idade. À esquerda estão os filtros de consulta que permitem uma manipulação mais detalhada dos dados para que eles sejam explorados de acordo com o interesse do usuário e à direita a descrição da ferramenta e o local de exibição da resposta. 


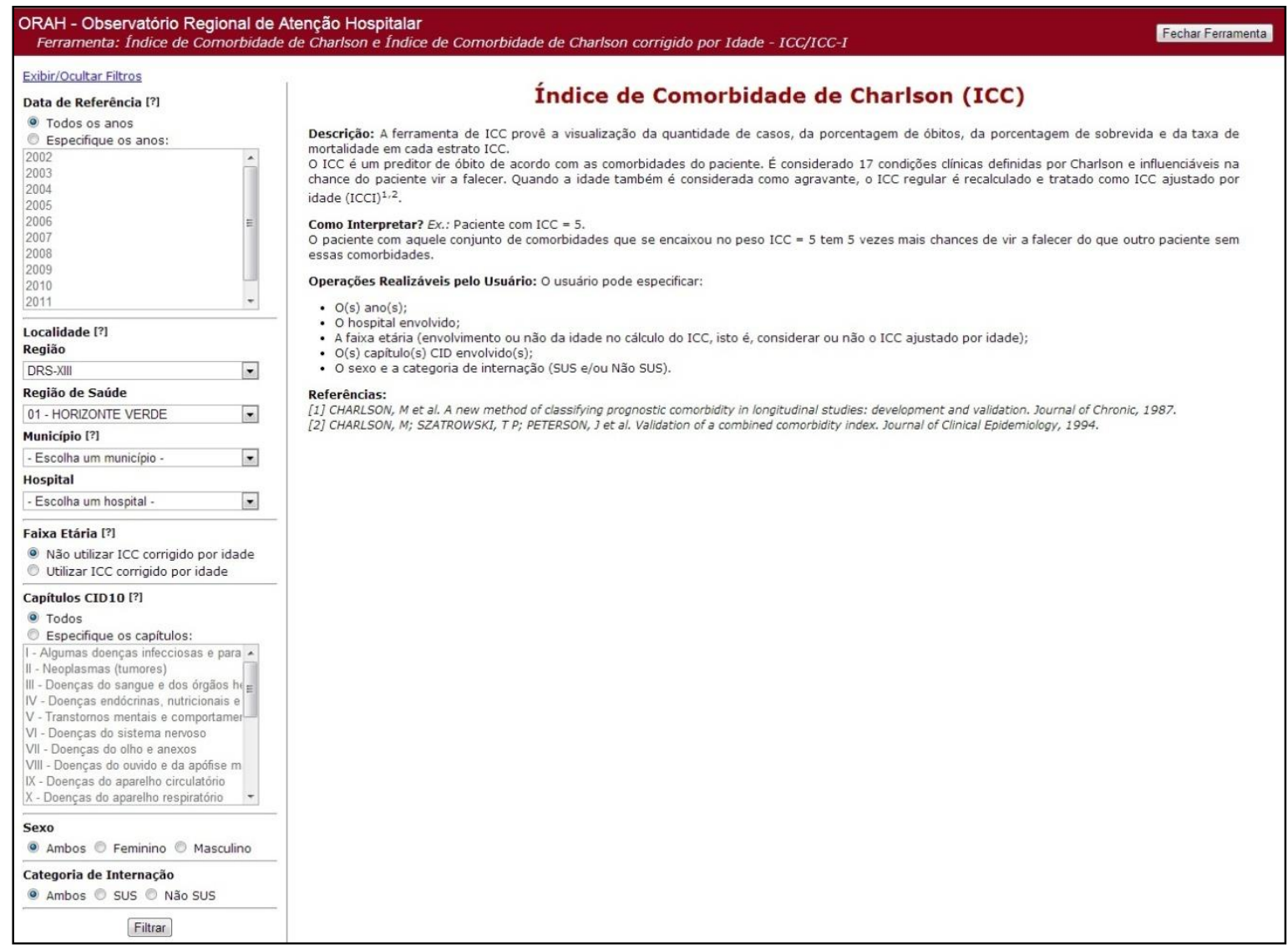

Figura 1- Interface inicial da ferramenta de ICC/ICCI incorporada ao portal web ORAH.

A Figura 2 é um exemplo de saída dos dados provenientes da ferramenta. A resposta apresenta uma tabela com o número de internações total, o número de internações cuja condição de saída se refere aos óbitos e o número de internações cuja condição de saída se refere às altas, em cada estrato ICC ou estrato ICCI. Podemos ainda, para facilitar a análise dos dados, com o auxílio dos botões apresentados abaixo da tabela, visualizar cada coluna de maneira gráfica (Figura 3). 


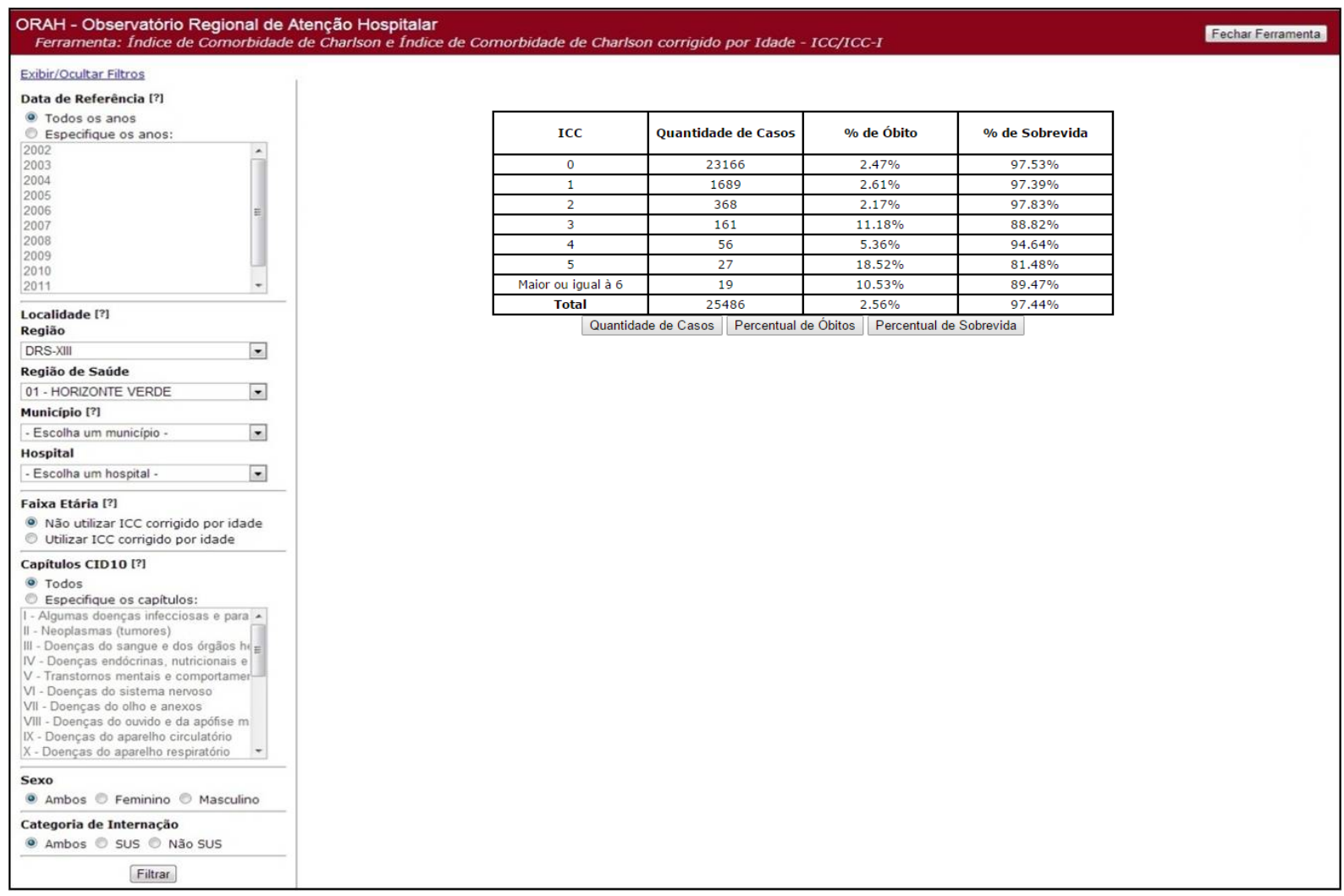

Figura 2 - Ferramenta ICC/ICCI alocada no Portal ORAH. Exemplo de saída dos dados - tabela.

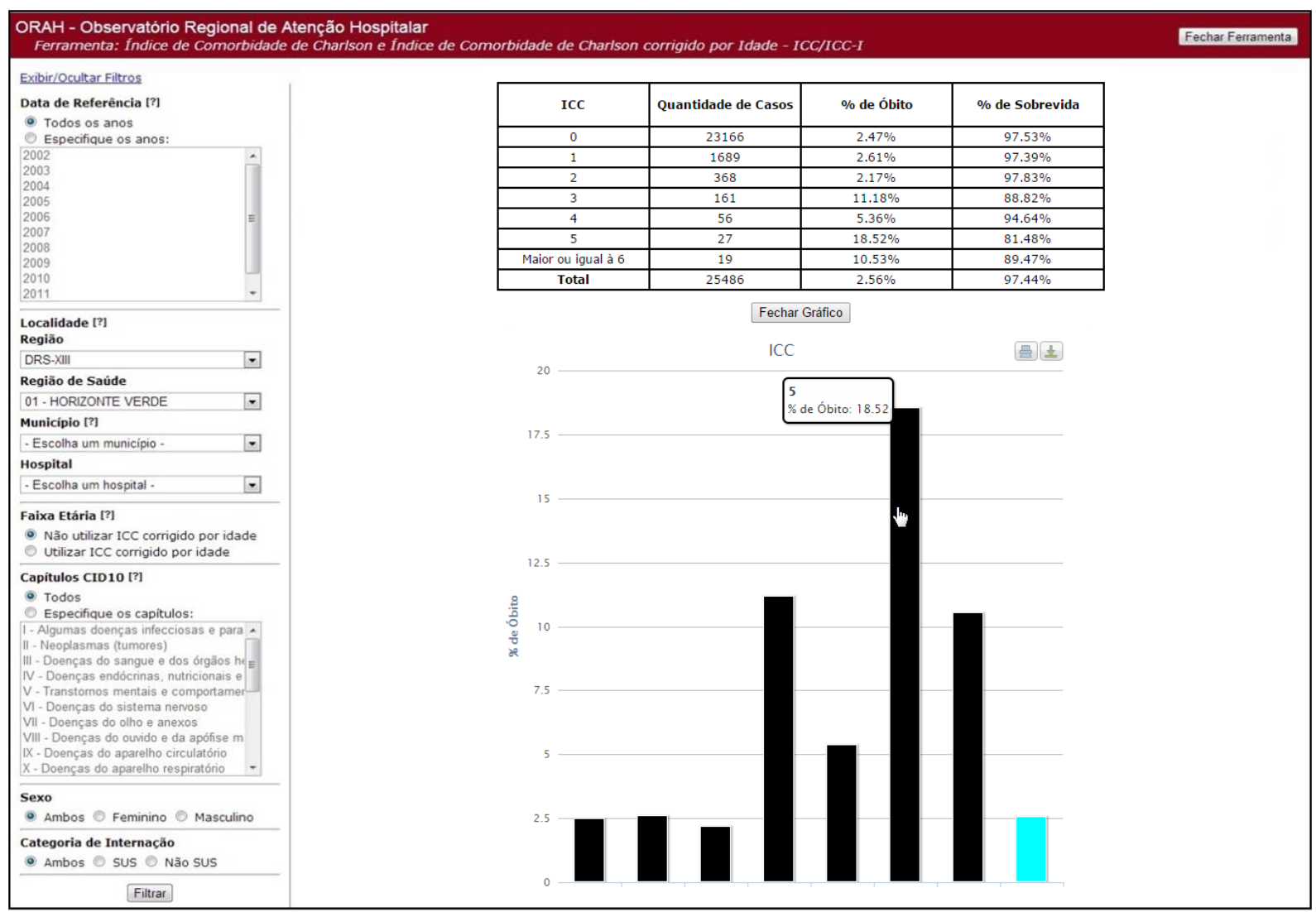

Figura 3 - Ferramenta ICC/ICCI alocada no Portal ORAH. Exemplo de saída dos dados - gráfico. 
Os achados expostos nas subseções seguintes foram obtidos com o auxílio da ferramenta descrita nessa subseção.

\subsubsection{CaracterizaÇão das Comorbidades}

Para o ano de 2011, nos 19 hospitais do estudo, tivemos 116.228 internações. A Tabela 3 expõe o número de internações de acordo com a quantidade de diagnósticos anotados nas quais 41.883 (36\%) apresentaram mais de um diagnóstico registrado.

Tabela 3 - Número de internações de acordo com a quantidade de diagnósticos anotados.

\begin{tabular}{c|c|c}
\hline \multirow{2}{*}{ Quantidade de diagnósticos anotados } & \multicolumn{2}{|c}{ Internações } \\
\cline { 2 - 3 } & $\boldsymbol{n}$ & $\boldsymbol{\%}$ \\
\hline Apenas 1 & 74.345 & 63,9 \\
\hline 2 & 20.235 & 17,4 \\
\hline 3 & 8.728 & 7,5 \\
\hline 4 & 4.658 & 4,1 \\
\hline 5 e mais & 8.262 & 7,1 \\
\hline
\end{tabular}

A fim de observarmos o comportamento das comorbidades no conjunto de dados e com que frequência as comorbidades listadas por Charlson aparecem no contexto, a Tabela 4 mostra as cinco comorbidades mais frequentes apresentadas pelos pacientes e o diagnóstico principal que mais se relacionou a elas.

Tabela 4 - As cinco comorbidades mais frequentes e o diagnóstico principal relacionado.

\begin{tabular}{c|c|c|c}
\hline \multicolumn{2}{|c|}{ Comorbidade } & \multicolumn{2}{c}{ Diagnóstico principal } \\
\hline CID 10 & Descrição & CID 10 & Descrição \\
\hline I10 & Hipertensão essencial (primária) & J189 & Pneumonia não especificada \\
\hline O829 & $\begin{array}{c}\text { Parto por cesariana não } \\
\text { especificada }\end{array}$ & O654 & $\begin{array}{c}\text { Obstrução do trabalho de parto devida a } \\
\text { anormalidades pélvicas maternas não } \\
\text { especificadas }\end{array}$ \\
\hline E149 & $\begin{array}{c}\text { Diabetes mellitus não especificado } \\
\text { - sem complicações }\end{array}$ & J189 & Pneumonia não especificada \\
\hline E788 & $\begin{array}{c}\text { Outros distúrbios do metabolismo } \\
\text { de lipoproteínas }\end{array}$ & I251 & Doença aterosclerótica do coração \\
\hline N390 & Cistite não especificada & J189 & Pneumonia não especificada \\
\hline
\end{tabular}

A hipertensão essencial (I10) foi a comorbidade mais frequente apresentada pelos pacientes que tinham em sua maioria, uma pneumonia não especificada (J189) como causa de internação. Nesses casos, pudemos perceber que essas doenças não são associadas entre si. Consideramos associação uma relação clínica entre os diagnósticos, ou seja, quando provavelmente um diagnóstico está relacionado diretamente na existência do outro. 
A segunda comorbidade mais frequente foi o parto por cesariana (O829) relacionado à causa de internação de obstrução do trabalho de parto (O654) sendo, nesse caso, a doença e o procedimento relacionados entre si.

Nota-se que apesar de termos um CID que representa o parto por cesariana, há um desconforto por percebermos que nessa situação o procedimento foi colocado em evidência nas comorbidades ao invés de serem relatadas outras condições clínicas do paciente, ou seja, essa anotação deveria estar vinculada aos procedimentos do paciente e não as comorbidades. Obviamente, os detalhes do porque desse achado foge do escopo desta pesquisa, mas podemos conjecturar que esse fato pode ser um possível viés de anotação do serviço ou do tratamento dos dados.

A Tabela 4 também nos mostra que dos diagnósticos selecionados por Charlson foram detectados como os mais frequentes na assistência hospitalar na região de Ribeirão Preto o diabetes mellitus (E149) e a pneumonia (doença pulmonar) (J189).

A Tabela 5 apresenta o número de internações de acordo com o tipo de atendimento (SUS e não SUS) e a quantidade de diagnósticos anotados. Os casos atendidos pelo SUS representaram $62 \%$ do total de internações. 30.165 (41,8\%) das internações atendidas pelo SUS apresentaram mais de um diagnóstico relacionado ao paciente, por outro lado, nas internações não atendidas pelo SUS apenas 11.688 (26,5\%) das internações apresentaram comorbidades anotadas. Do conjunto estudado, 37 casos - menos de 0,1\% - não foram classificados nessa análise pois estavam com o valor da variável tipo de atendimento prejudicado (nulo).

Tabela 5 - Número de internações de acordo com o tipo de atendimento (SUS e não SUS) e a quantidade de diagnósticos anotados.

\begin{tabular}{c|c|c|c|c}
\hline \multirow{2}{*}{ Quantidade de diagnósticos } & \multicolumn{4}{|c}{ Internações } \\
\cline { 2 - 5 } & \multicolumn{2}{|c}{ SUS } & \multicolumn{1}{c}{ não SUS } \\
\cline { 2 - 5 } & $\boldsymbol{n}$ & $\%$ & $\boldsymbol{n}$ & $\%$ \\
\hline Apenas 1 & 41.945 & 36,1 & 32.393 & 27,9 \\
\hline 2 & 12.252 & 10,5 & 7.983 & 6,9 \\
\hline 3 & 6.290 & 5,4 & 2.438 & 2,1 \\
\hline 4 & 3.784 & 3,3 & 844 & 0,7 \\
\hline 5 e mais & 7.839 & 6,7 & 423 & 0,4 \\
\hline
\end{tabular}

As comorbidades mais frequentes e a causa de internação mais comum relacionadas a elas estão listadas na Tabela 6 para os atendimentos SUS e na Tabela 7 para os atendimentos não SUS.

A hipertensão essencial (I10) e o parto por cesárea não especificado (O829) aparecem em ambos os serviços. Nos atendimentos SUS as internações por doença aterosclerótica do 
coração (I251) mostraram com maior frequência comorbidades relacionadas ao paciente. Já nos atendimentos não SUS as internações por pneumonia não especificada (J189) apresentaram maior frequência de comorbidades ao paciente.

Tabela 6 - As cinco comorbidades mais frequentes e o diagnóstico principal mais comum relacionado segundo atendimento SUS.

\begin{tabular}{c|c|c|c}
\hline \multicolumn{2}{|c|}{ Comorbidade } & \multicolumn{2}{|c}{ Diagnóstico principal mais comum } \\
\hline CID 10 & Descrição & CID 10 & Descrição \\
\hline I10 & Hipertensão essencial (primária) & I678 & $\begin{array}{c}\text { Outras doenças cerebrovasculares } \\
\text { especificadas }\end{array}$ \\
\hline E788 & $\begin{array}{c}\text { Outros distúrbios do metabolismo de } \\
\text { lipoproteínas }\end{array}$ & I251 & Doença aterosclerótica do coração \\
\hline F172 & $\begin{array}{c}\text { Transtornos mentais e comportamentais } \\
\text { devidos ao uso de fumo - síndrome de } \\
\text { dependência }\end{array}$ & I251 & Doença aterosclerótica do coração \\
\hline E119 & $\begin{array}{c}\text { Diabetes mellitus não-insulino- } \\
\text { dependente - sem complicações }\end{array}$ & I251 & Doença aterosclerótica do coração \\
\hline O829 & Parto por cesariana não especificada & O689 & $\begin{array}{c}\text { Trabalho de parto e parto complicados por } \\
\text { sofrimento fetal, não especificado }\end{array}$ \\
\hline
\end{tabular}

Tabela 7 - As cinco comorbidades mais frequentes e o diagnóstico principal mais comum relacionado segundo atendimento não SUS.

\begin{tabular}{c|c|c|c}
\hline \multicolumn{2}{|c|}{ Comorbidade } & \multicolumn{2}{|c}{ Diagnóstico principal mais comum } \\
\hline CID 10 & Descrição & CID 10 & Descrição \\
\hline O829 & Parto por cesariana não especificada & O654 & $\begin{array}{c}\text { Obstrução do trabalho de parto devida a } \\
\text { anormalidades pélvicas maternas não } \\
\text { especificadas }\end{array}$ \\
\hline I10 & Hipertensão essencial (primária) & J189 & Pneumonia não especificada \\
\hline E149 & $\begin{array}{c}\text { Diabetes mellitus não especificado - } \\
\text { sem complicações }\end{array}$ & J189 & Pneumonia não especificada \\
\hline J189 & Pneumonia não especificada & A419 & Septicemia não especificada \\
\hline N390 & Cistite não especificada & J189 & Pneumonia não especificada \\
\hline
\end{tabular}

Quando apresentamos os casos por tipo de atendimento verificamos mais duas condições clínicas selecionadas por Charlson, outras doenças cerebrovasculares especificadas (I678) e doença aterosclerótica do coração que também são frequentes nos casos do estudo.

Tabela 8 - Número de internações de acordo com a faixa etária do paciente e a quantidade de diagnósticos anotados.

\begin{tabular}{|c|c|c|c|c|c|c|c|c|c|c|c|c|}
\hline \multirow{3}{*}{$\begin{array}{l}\text { Quantidade de } \\
\text { diagnósticos }\end{array}$} & \multicolumn{12}{|c|}{ Internações } \\
\hline & \multicolumn{2}{|c|}{$\begin{array}{c}\text { Menor que } 50 \\
\text { anos }\end{array}$} & \multicolumn{2}{|c|}{$50-59$ anos } & \multicolumn{2}{|c|}{ 60-69 anos } & \multicolumn{2}{|c|}{ 70-79 anos } & \multicolumn{2}{|c|}{ 80-89 anos } & \multicolumn{2}{|c|}{$\begin{array}{c}\text { Maior que } 90 \\
\text { anos }\end{array}$} \\
\hline & $n$ & $\%$ & $n$ & $\%$ & $n$ & $\%$ & $n$ & $\%$ & $n$ & $\%$ & $n$ & $\%$ \\
\hline Apenas 1 & 47.538 & 40,9 & 8.859 & 7,6 & 7.398 & 6,4 & 5902 & 5,1 & 3.856 & 3,3 & 785 & 0,7 \\
\hline 2 & 11.052 & 9,5 & 2.796 & 2,4 & 2.528 & 2,2 & 2.166 & 1,9 & 1.370 & 1,2 & 323 & 0,3 \\
\hline 3 & 3.886 & 3,3 & 1.293 & 1,1 & 1.392 & 1,2 & 1.215 & 1,0 & 790 & 0,7 & 152 & 0,1 \\
\hline 4 & 1.984 & 1,7 & 727 & 0,6 & 818 & 0,7 & 657 & 0,6 & 402 & 0,3 & 70 & 0,1 \\
\hline 5 e mais & 4.030 & 3,5 & 1.282 & 1,1 & 1.232 & 1,1 & 1.059 & 0,9 & 563 & 0,5 & 96 & 0,1 \\
\hline
\end{tabular}


A Tabela 8 (página 33) mostra o número de internações de acordo com a faixa etária do paciente e a quantidade de diagnósticos anotados. As internações de pacientes com idade maior que 50 anos representam $41 \%$ do total de internações e para essas internações apenas $18 \%$ apresentavam mais que uma comorbidade anotada. Para a variável faixa etária, apenas sete casos estavam com o valor da variável prejudicado (nulo) e não foram classificados.

A Tabela 9 apresenta as três comorbidades mais frequentes e o diagnóstico principal mais comum relacionado a elas de acordo com a faixa etária dos pacientes internados.

Tabela 9 - As três comorbidades mais frequentes e o diagnóstico mais comum relacionado segundo faixa etária.

\begin{tabular}{|c|c|c|c|c|}
\hline \multirow{2}{*}{$\begin{array}{l}\text { Faixa } \\
\text { Etária }\end{array}$} & \multicolumn{2}{|r|}{ Comorbidade } & \multicolumn{2}{|r|}{ Diagnóstico principal mais comum } \\
\hline & CID 10 & Descrição & CID 10 & Descrição \\
\hline \multirow{3}{*}{$\begin{array}{l}\text { Menor que } \\
50 \text { anos }\end{array}$} & $\mathrm{I} 10$ & $\begin{array}{l}\text { Hipertensão essencial } \\
\text { (primária) }\end{array}$ & $\mathrm{I} 200$ & Angina instável \\
\hline & O829 & $\begin{array}{l}\text { Parto por cesariana não } \\
\text { especificada }\end{array}$ & O654 & $\begin{array}{c}\text { Obstrução do trabalho de parto devida a } \\
\text { desproporção feto-pélvica, não } \\
\text { especificada }\end{array}$ \\
\hline & E149 & $\begin{array}{l}\text { Diabetes mellitus não } \\
\text { especificado - sem } \\
\text { complicações }\end{array}$ & I219 & $\begin{array}{l}\text { Infarto agudo do miocárdio não } \\
\text { especificado }\end{array}$ \\
\hline \multirow{3}{*}{$\begin{array}{c}\text { De } 50 \text { a } 59 \\
\text { anos }\end{array}$} & $\mathrm{I} 10$ & $\begin{array}{l}\text { Hipertensão essencial } \\
\text { (primária) }\end{array}$ & I200 & Angina instável \\
\hline & E788 & $\begin{array}{c}\text { Outros distúrbios do } \\
\text { metabolismo de lipoproteínas }\end{array}$ & $\mathrm{I} 251$ & Doença aterosclerótica do coração \\
\hline & E149 & $\begin{array}{c}\text { Diabetes mellitus não } \\
\text { especificado - sem } \\
\text { complicações }\end{array}$ & 1200 & Angina instável \\
\hline \multirow{3}{*}{$\begin{array}{c}\text { De } 60 \text { a } 69 \\
\text { anos }\end{array}$} & $\mathrm{I} 10$ & $\begin{array}{l}\text { Hipertensão essencial } \\
\text { (primária) }\end{array}$ & $\mathrm{I} 251$ & Doença aterosclerótica do coração \\
\hline & E149 & $\begin{array}{l}\text { Diabetes mellitus não } \\
\text { especificado - sem } \\
\text { complicações }\end{array}$ & J189 & Pneumonia não especificada \\
\hline & E788 & $\begin{array}{c}\text { Outros distúrbios do } \\
\text { metabolismo de lipoproteínas }\end{array}$ & $\mathrm{I} 251$ & Doença aterosclerótica do coração \\
\hline \multirow{3}{*}{$\begin{array}{c}\text { De } 70 \text { a } 79 \\
\text { anos }\end{array}$} & $\mathrm{I} 10$ & $\begin{array}{c}\text { Hipertensão essencial } \\
\text { (primária) }\end{array}$ & J189 & Pneumonia não especificada \\
\hline & E149 & $\begin{array}{l}\text { Diabetes mellitus não } \\
\text { especificado - sem } \\
\text { complicações }\end{array}$ & J189 & Pneumonia não especificada \\
\hline & E788 & $\begin{array}{l}\text { Outros distúrbios do } \\
\text { metabolismo de lipoproteínas }\end{array}$ & $\mathrm{I} 251$ & Doença aterosclerótica do coração \\
\hline \multirow{3}{*}{$\begin{array}{c}\text { De } 80 \text { a } 89 \\
\text { anos }\end{array}$} & $\mathrm{I} 10$ & $\begin{array}{l}\text { Hipertensão essencial } \\
\text { (primária) }\end{array}$ & J189 & Pneumonia não especificada \\
\hline & J189 & Pneumonia não especificada & I500 & Insuficiência cardíaca congestiva \\
\hline & E149 & $\begin{array}{l}\text { Diabetes mellitus não } \\
\text { especificado - sem } \\
\text { complicações } \\
\end{array}$ & J189 & Pneumonia não especificada \\
\hline \multirow{3}{*}{$\begin{array}{l}\text { Maior que } \\
90 \text { anos }\end{array}$} & $\mathrm{I} 10$ & $\begin{array}{l}\text { Hipertensão essencial } \\
\text { (primária) }\end{array}$ & J189 & Pneumonia não especificada \\
\hline & N390 & Cistite não especificada & J189 & Pneumonia não especificada \\
\hline & J189 & Pneumonia não especificada & N390 & Cistite não especificada \\
\hline
\end{tabular}


Apresentando os casos por faixa etária, a hipertensão essencial (I10) foi a comorbidade mais frequente observada e a partir dos 50 anos tivemos prioritariamente como causas de internações doenças pertencentes aos Capítulos IX (Doenças do aparelho circulatório) e X (Doenças do aparelho respiratório) da CID 10.

\subsubsection{Caracterização da Distribuição de Risco de Óbito CoM}

\section{BASE NO ICC/ICCI}

\subsubsection{VISÃo Geral}

A Tabela 10 lista as 17 condições clínicas estudadas por Charlson, suas pontuações de acordo com o ICC e o número de internações de cada uma das comorbidades estudadas. 5.760 casos fazem parte do conjunto de internações com condições clínicas selecionadas por Charlson representando 5\% do total de internações dos hospitais estudados para o ano de 2011.

Tabela 10 - Frequência das condições clínicas estudadas por Charlson.

\begin{tabular}{|c|c|c|c|c|c|}
\hline \multicolumn{2}{|r|}{ Condição Clínica } & \multicolumn{4}{|c|}{ Internações } \\
\hline \multirow{2}{*}{ Pontuação } & \multirow{2}{*}{ Descrição } & \multicolumn{2}{|c|}{ Por comorbidade } & \multicolumn{2}{|c|}{ Por estrato } \\
\hline & & $n$ & $\%$ & $n$ & $\%$ \\
\hline 1 & Infarto Agudo do Miocárdio & 356 & 0,31 & \multirow{10}{*}{4659} & \multirow{10}{*}{4,01} \\
\hline 1 & Insuficiência Cardíaca Congestiva & 0 & 0 & & \\
\hline 1 & Doença Vascular-Periférica & 190 & 0,16 & & \\
\hline 1 & Acidente Vascular Cerebral (AVC) & 415 & 0,36 & & \\
\hline 1 & Demência & 10 & 0,01 & & \\
\hline 1 & Doença Pulmonar & 178 & 0,15 & & \\
\hline 1 & Doença do tecido conjuntivo & 80 & 0,07 & & \\
\hline 1 & Úlcera & 0 & 0 & & \\
\hline 1 & Doença hepática & 316 & 0,27 & & \\
\hline 1 & Diabetes sem complicações & 3114 & 2,68 & & \\
\hline 2 & Diabetes com complicações & 357 & 0,31 & \multirow{4}{*}{645} & \multirow{4}{*}{0,55} \\
\hline 2 & Hemiplegia ou paraplegia & 9 & 0,01 & & \\
\hline 2 & Doença renal severa ou moderada & 135 & 0,12 & & \\
\hline 2 & Câncer & 144 & 0,12 & & \\
\hline 3 & Câncer em metástase & 112 & 0,10 & \multirow{2}{*}{394} & \multirow{2}{*}{0,34} \\
\hline 3 & Doença do fígado moderada à grave & 282 & 0,24 & & \\
\hline 6 & AIDS & 62 & 0,05 & 62 & 0,05 \\
\hline
\end{tabular}

A Figura 4 exibe a quantidade de casos por estrato ICC. Desconsiderando o estrato 0 sem comorbidades referidas ao paciente - os estratos 1 e 2, de menor risco de óbito, apresentam o maior número de casos. 


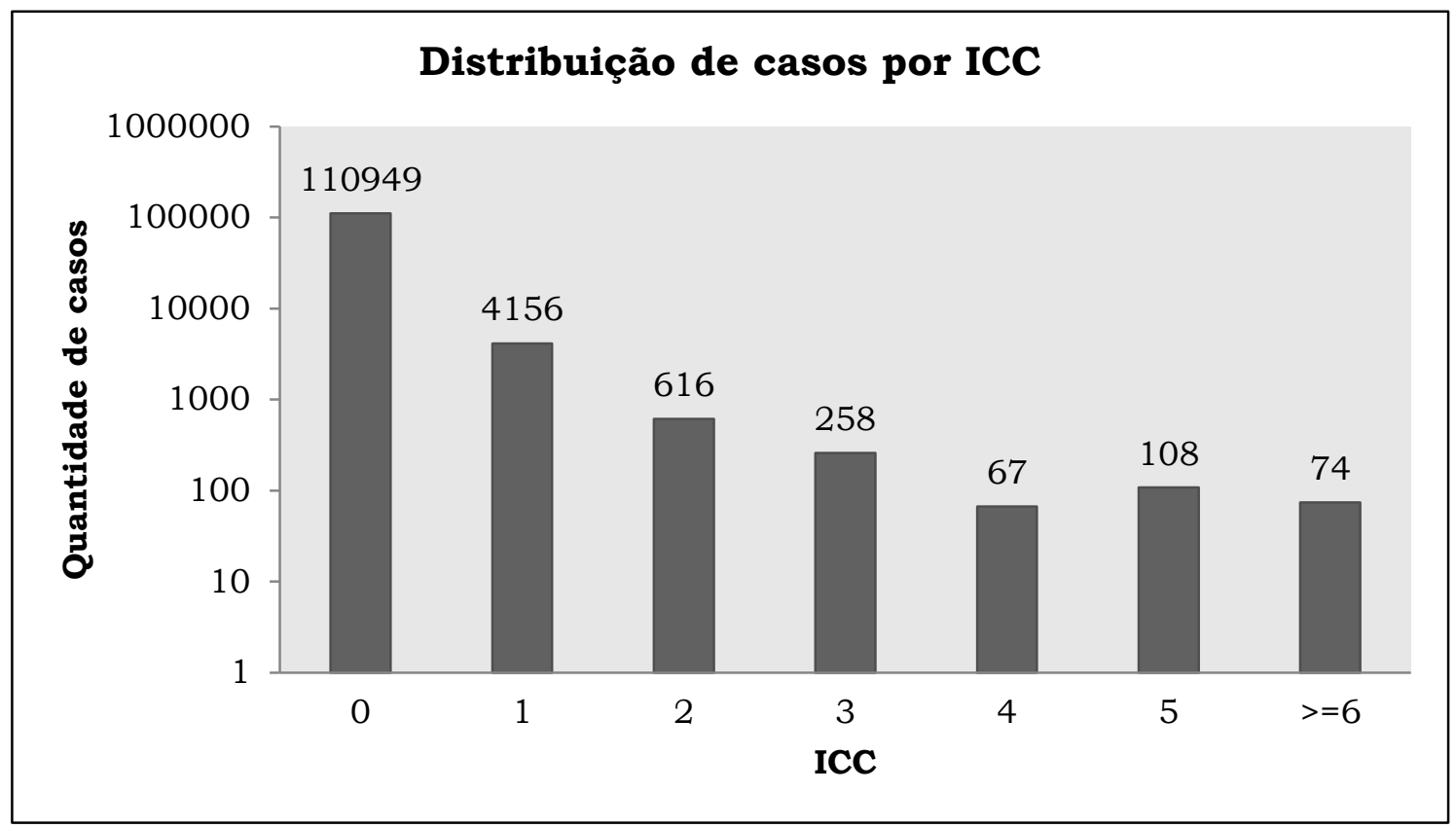

Figura 4 - Gráfico de distribuição de casos por estrato ICC.

De acordo com a Figura 5, pudemos observar que apesar dos estratos com valores altos (maiores que 3) do ICC apresentarem as maiores taxas de óbitos do conjunto, a curva de mortalidade não é crescente e não se alinha a distribuição de risco prevista por Charlson cujas maiores pontuações do ICC sugerem maiores riscos de óbito e consequentemente devem resultar em maiores taxas de óbito. No entanto, a pesquisa não visa investigar o porquê do comportamento da curva, que pode ser uma característica do conjunto de dados trabalhado ou então um reflexo do cuidado prestado aos pacientes nessa região. Percebemos, então, que nesse conjunto, as internações classificadas nos estratos 4 e 5 representam os casos mais graves. 


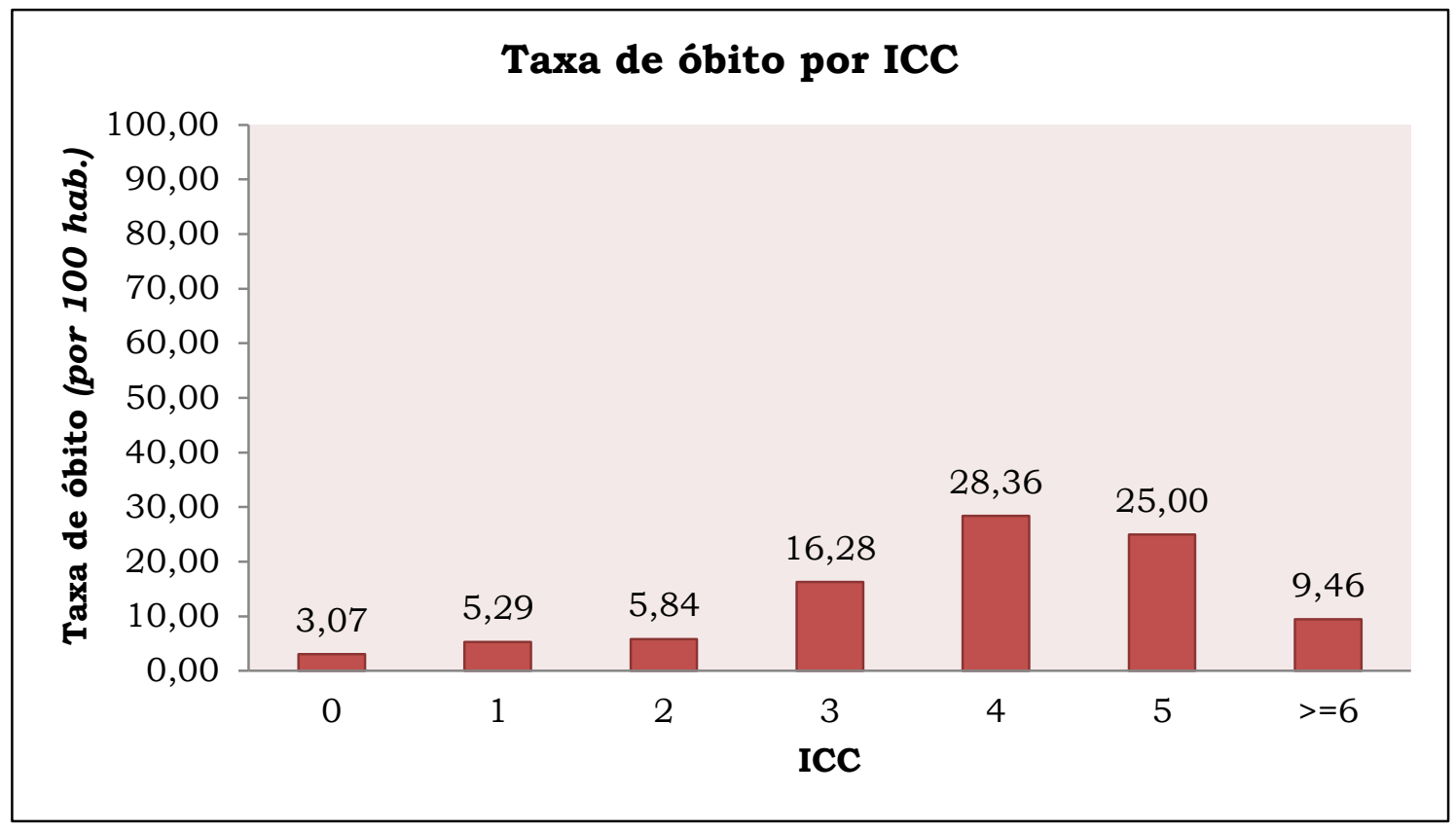

Figura 5 - Gráfico de taxa de óbito por estrato ICC.

A Figura 6 exibe a quantidade de casos distribuídos por estrato ICCI. Os estratos 1,2 e 3 apresentam o maior número de casos.

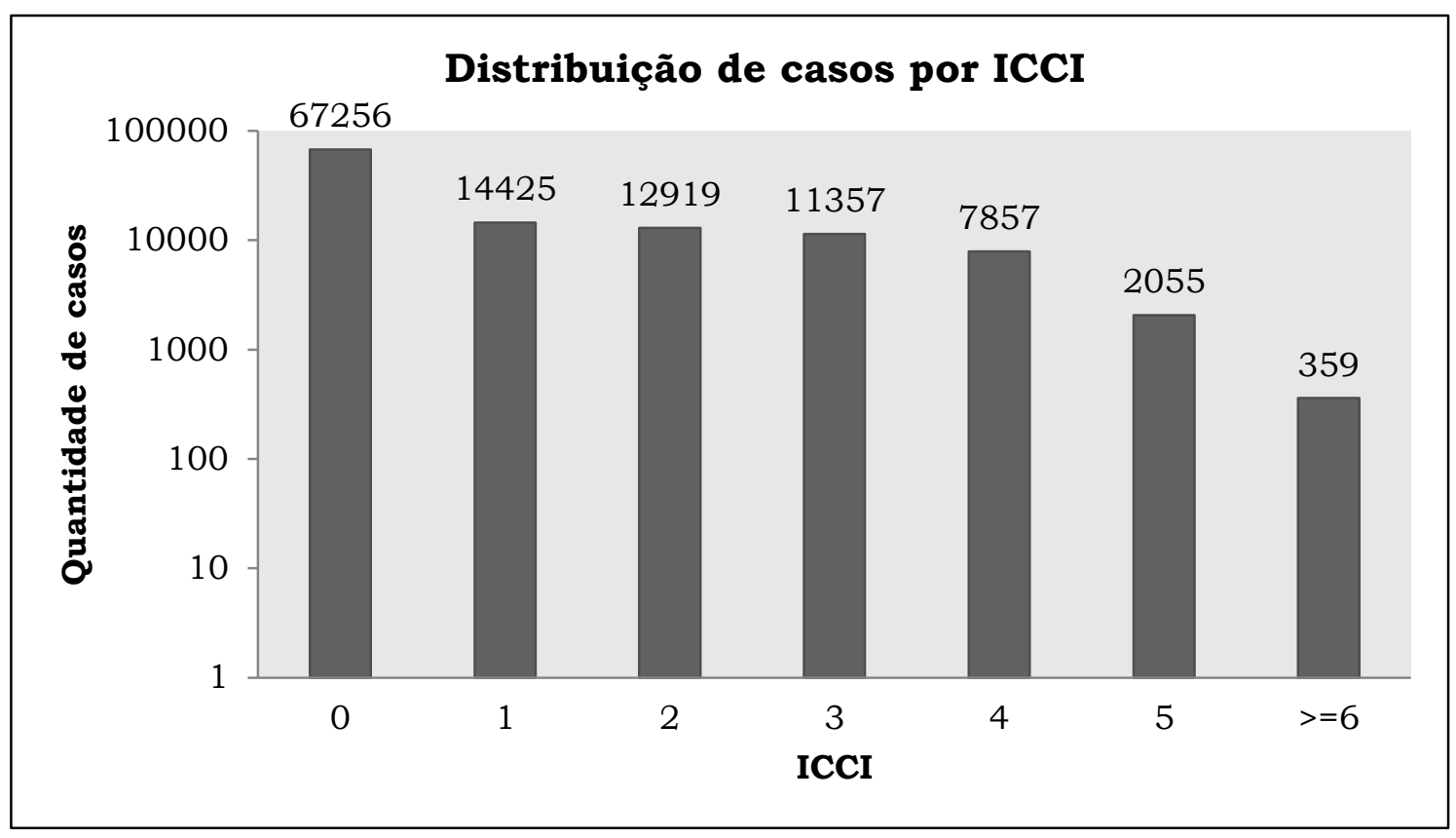

Figura 6 - Gráfico de distribuição de casos por estrato ICCI.

De acordo com a Figura 7, pode-se observar que para o ICCI a curva de mortalidade do conjunto é crescente e se alinha a distribuição de risco prevista por Charlson, sendo assim, o ICCI tornou-se mais apropriado para estudarmos a gravidade desses casos. Nesse conjunto, as internações alocados nos estratos 5 e 6 representam os casos mais graves. 


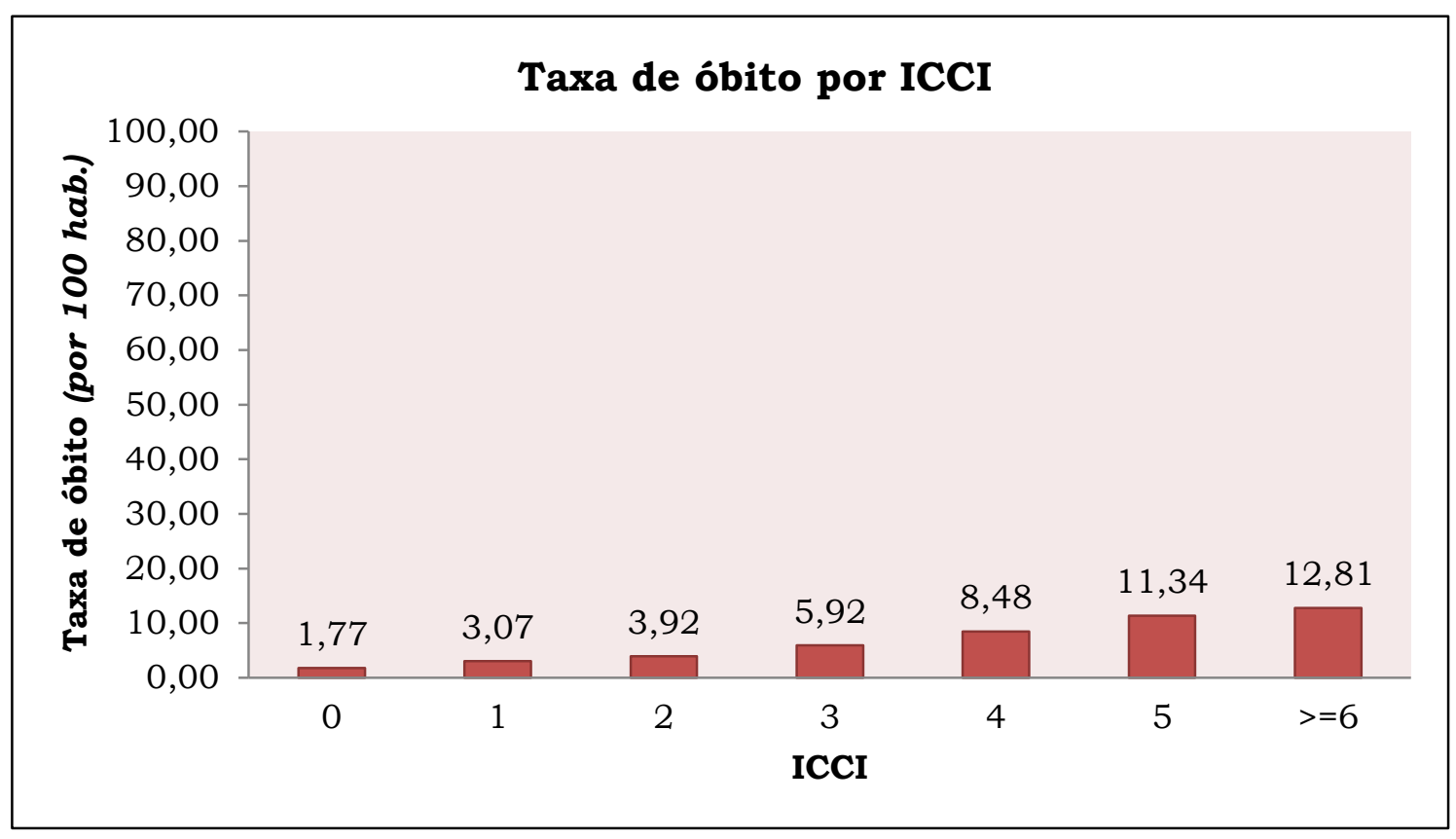

Figura 7 - Gráfico de taxa de óbito por estrato ICCI.

As Figuras 8 e 9 exibem a relação das taxas de óbito de acordo com o tipo de atendimento. Essa relação é expressa pela taxa de óbito das internações atendidas pelo SUS dividido pela taxa de óbito dos atendimentos não SUS, em cada estrato ICC e ICCI.

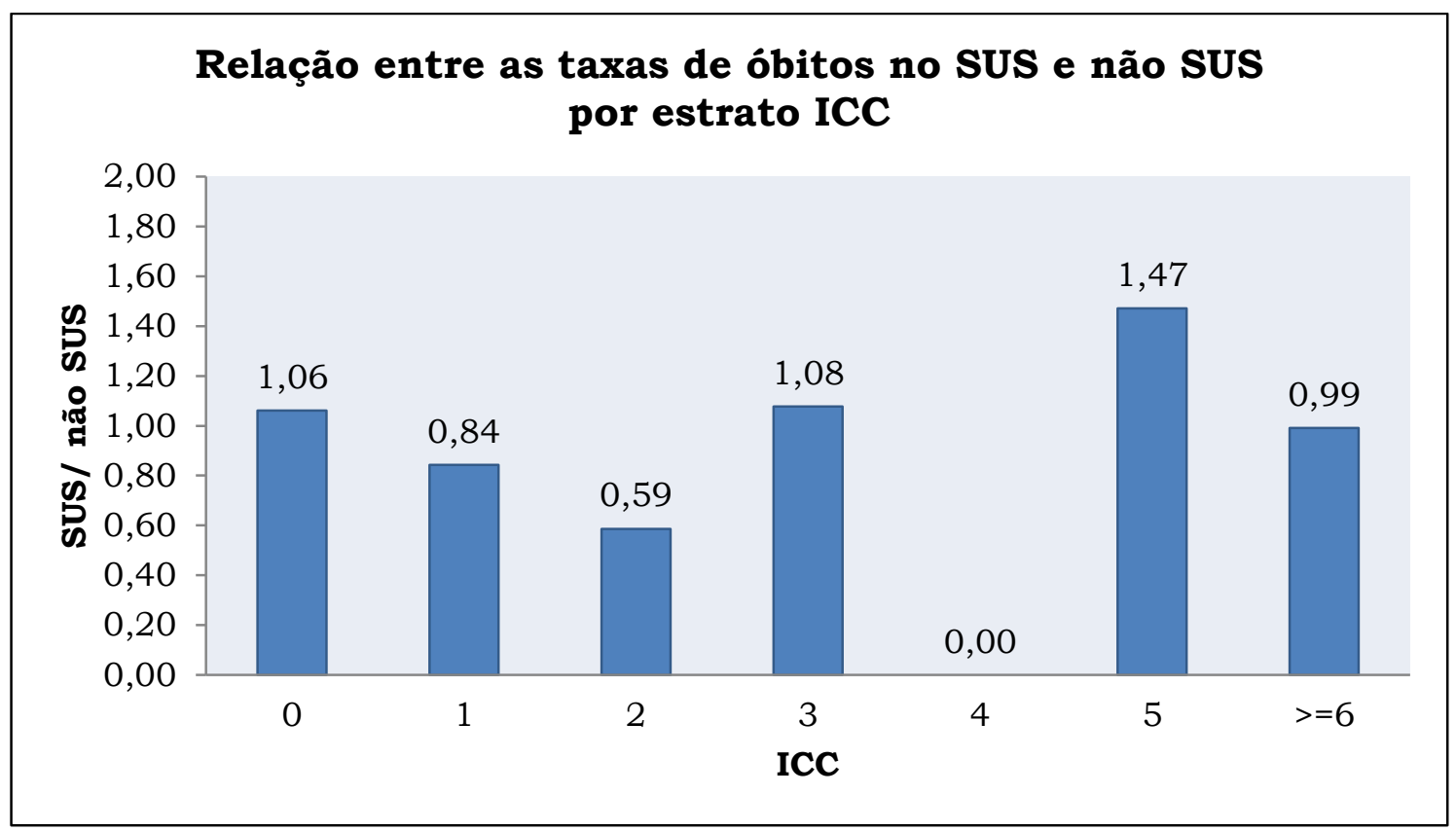

Figura 8 - Gráfico de relação entre as taxas de óbitos com o tipo de atendimento por estrato ICC. 


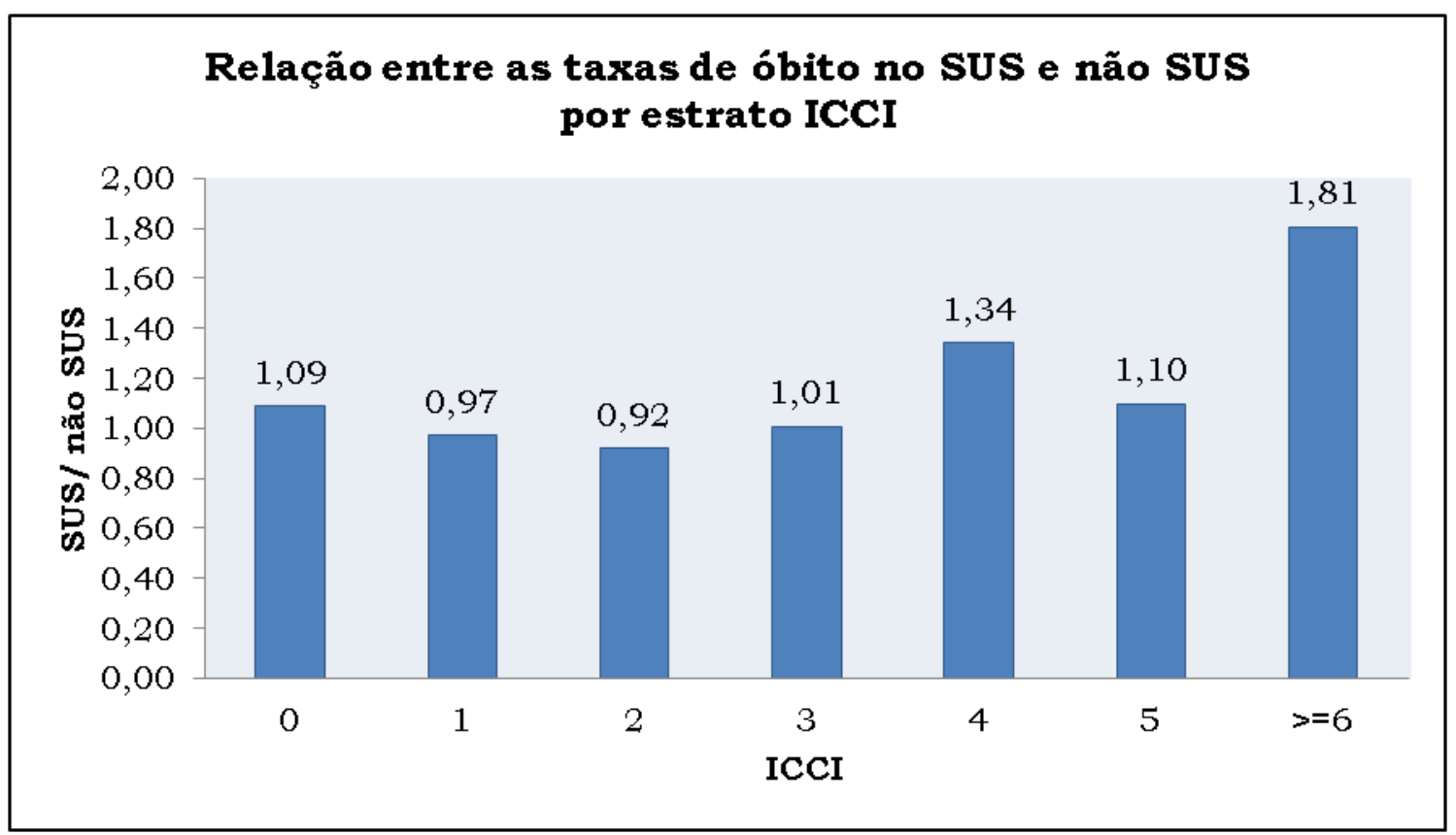

Figura 9 - Gráfico da relação entre as taxas de óbito com o tipo de atendimento por estrato ICCI.

Diante disso, percebemos que, por ICCI, os casos atendidos pelo SUS foram os casos de maior risco de óbito, isto é, a relação nos estratos de alta pontuação (estratos 4,5 e 6) é maior que 1. Já de acordo com o ICC não pudemos ver esse regularidade nos estratos de alta pontuação e isso sugere uma forte associação da variável idade nos casos estudados em relação ao comportamento da curva de óbito.

\subsubsection{DISTRIBUIÇÃO ESPACIAL DE ACORDO COM A GRAVIDADE DOS CASOS}

A distribuição de gravidade dos casos foi baseada nos cálculos de ICCI desse conjunto devido a taxa de óbito ter se alinhado a definição prevista por Chalson. A Figura 10 mostra a divisão entre os casos de baixa gravidade (a) e alta gravidade (b). 


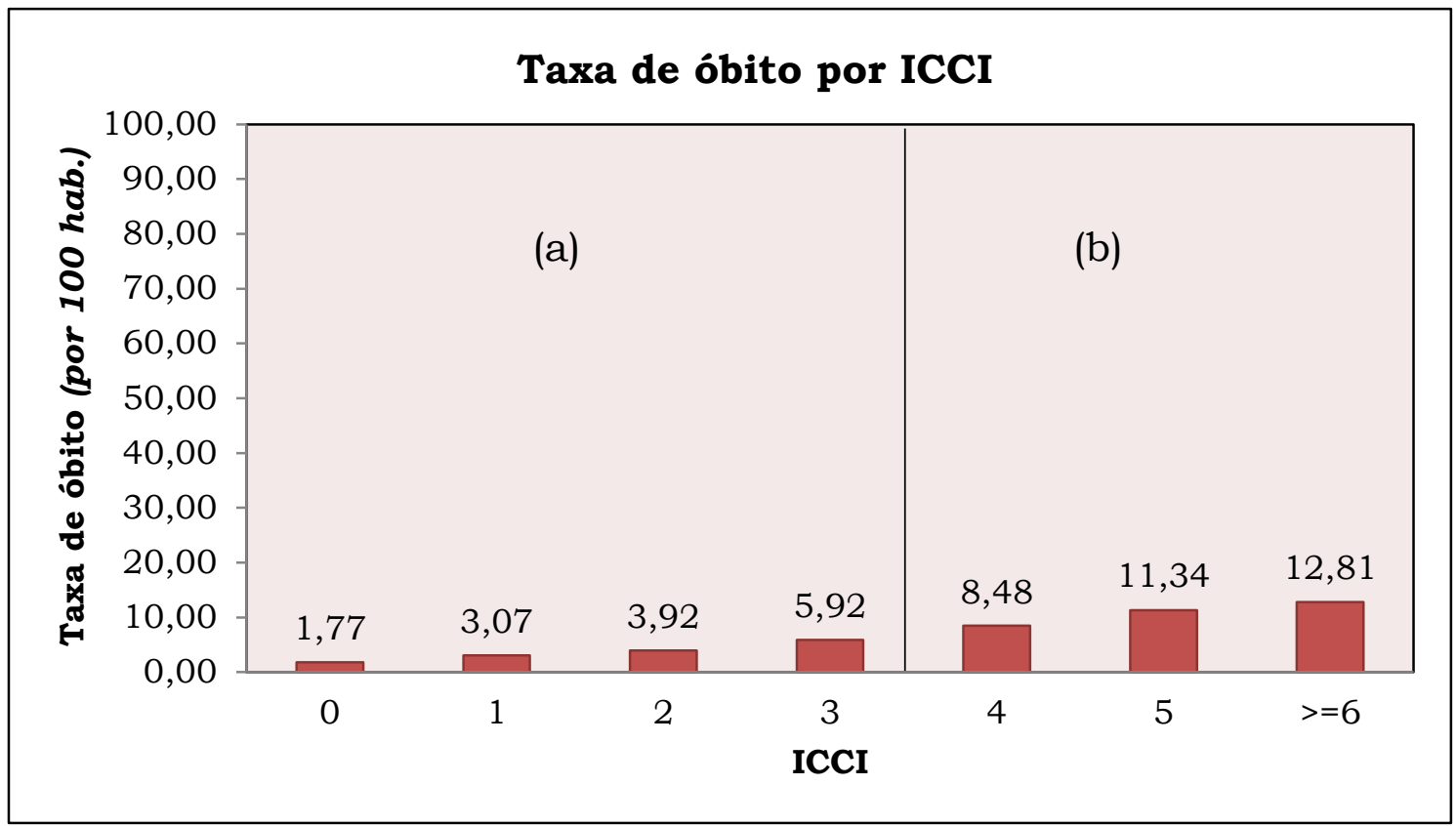

Figura 10 - Gráfico de taxa de óbito por estrato ICCI para apresentar a gravidade dos casos: (a) Casos de baixa gravidade e (b) Casos de alta gravidade.

Com isso, separamos, por hospital, os casos de alta gravidade - com pontuação ICCI maior ou igual a 4 - e dividimos pelo total de atendimentos (Figura 11). Pudemos perceber que as cidades de Ribeirão Preto, Sertãozinho, Cajuru e Jaboticabal foram as que assistiram a maior quantidade de casos de alta gravidade em 2011. 


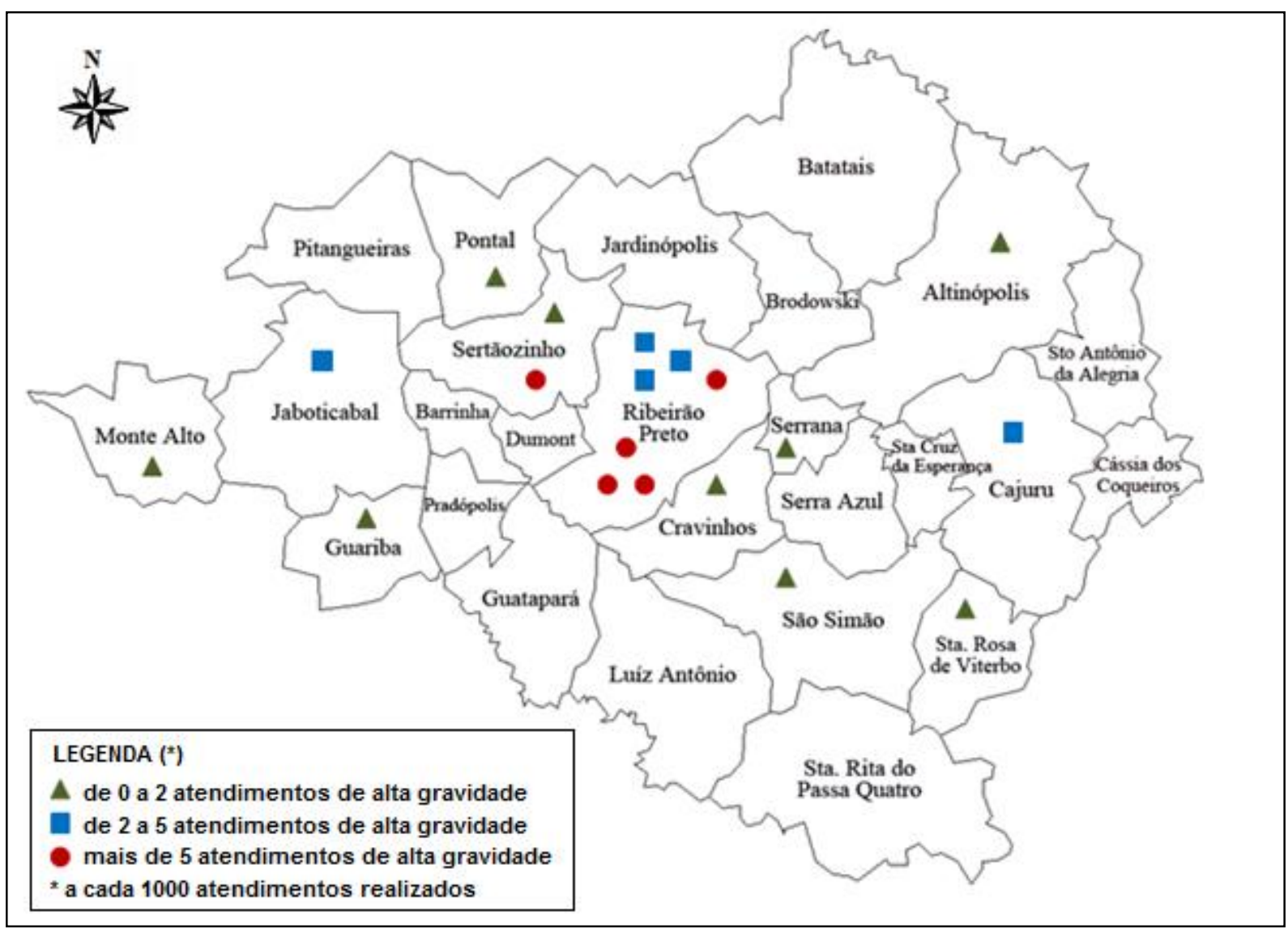

Figura 11 - Representação espacial dos casos de alta gravidade.

A distribuição dos atendimentos dos casos de alta gravidade segue o príncipio da Regionalização, uma diretriz do Sistema Único de Saúde e um eixo estruturante do Pacto de Gestão. A Regionalização visa a estruturação dos serviços de saúde e prevê que cidades pólos em assistência devem atrair maior quantidade de casos e oferecer serviços especializados em maior escala. A cidade de Ribeirão Preto - pertencente a Região de Saúde do Aquífero Guarani e sede do Departamento Regional de Saúde XIII - é pólo de saúde para a região, pertence a uma área de urbanização complexa onde há fluxos de pacientes intensos e volumos e com serviços hospitalares capazes de assistir pacientes de toda região. As cidades de Sertãozinho, Jaboticabal e Cajuru - pertencentes as Regiões de Saúde do Horizonte Verde e Vale das Cachoeiras - são considerados municípios sub-pólos na região, pois concentram serviços capazes de atrair pacientes originados de municípios ao seu entorno e, assim, compartem com o pólo a atenção as demandas (UNICAMP; MINISTÉRIO DA SAÚDE, 2002). 


\subsubsection{TRABALHOS APRESENTADOS EM EVENTOS}

Alguns resultados desse trabalho foram expostos em congressos nacionais e internacionais permitindo a discussão e a troca de experiências no assunto.

A primeira exposição foi no Congresso Brasileiro de Informática em Saúde - 2012 (HITOMI et al., 2012) que apresentava a ferramenta computacional de automatização do cálculo do Índice de Comorbidade de Charlson ainda em desenvolvimento. Após as fases de desenvolvimento, adaptação e incorporação, a ferramenta foi apresentada, então, junto ao conjunto de ferramentas do portal ORAH no XIII Workshop de Informática Médica - 2013 (CARVALHO et al., 2013a).

Com o intuito de discutir sobre o perfil das internações ocorridas em Ribeirão Preto, foi apresentado no $10^{\circ}$ Congresso Brasileiro de Saúde Coletiva - 2012 um estudo da gravidade dos casos nos anos de 2007 e 2008 com base no ICCI, considerando o valor do índice, a distribuição por sexo e por condição de saída (óbito ou alta) (CARVALHO et al., 2012) e também foi apresentado no Congresso Ibero-Americano de Epidemiologia e Saúde Pública um estudo sobre a predição de óbito das internações com base no ICC considerando o valor do índice e do tipo de atendimento (público ou privado) (CARVALHO et al., 2013b).

Por fim, um retrato das comorbidades mais frequentes ao longo de 10 anos em nossa região foi discutido com a apresentação de um estudo no IX Congresso Brasileiro de Epidemiologia (CARVALHO et al., 2014). 


\subsection{CONCLUSÃo}

O trabalho visou identificar a distribuição do risco de óbito com a análise das comorbidades relacionadas ao paciente. O ICC puro, originalmente, surgiu para identificar a gravidade dos casos em hospitais de grande porte para a cidade de Nova Iorque, uma realidade hospitalar diferente da encontrada em nossa região. Quando retratamos nossos dados por meio do ICC puro, a mortalidade não se comportou de maneira crescente, não sugerindo um alto risco nos estratos com maior pontuação. Apesar disso, quando analisamos as distribuições com o ICCI, a mortalidade se comportou da maneira prevista por Charlson, de forma crescente, onde os casos mais graves nos dava uma maior taxa de óbito, indicando, assim, que na assistência prestada na região a idade é um importante fator de associação com o risco de óbito.

A ferramenta disponibilizada em um portal web de conteúdo permitindo o manuseio em diferentes estratos e situações - de acordo com sexo, diagnóstico principal, tipo de atendimento, entre outros - oferece aos usuários o diagnóstico da gravidade dos casos que estão sendo atendidos por aquele hospital, quais comorbidades dentre as listadas por Charlson merecem mais atenção no cuidado e qual a taxa de óbito em relação aos casos de baixa e alta gravidade. Diante disso, pudemos perceber que o indicador é um bom auxílio aos gestores para análise do cuidado prestado possibilitando o levantamento de ações para a melhoria do mesmo. 


\subsection{CONSIDERACÕES FINAIS}

O Índice de Comorbidade de Charlson é pouco explorado na literatura e no decorrer do trabalho percebemos que o estudo do índice auxilia na tomada de decisões e no entendimento dos cuidados relacionados à saúde. Como trabalho futuro, a automatização do cálculo e os dados disponibilizados pelo CPDH podem auxiliar na construção de um vetor de comorbidades com base no nosso cenário e tornando assim, as análises da distribuição do risco de óbito mais fiel a situação de saúde de nossa região. 


\section{REFERÊNCIAS}

ALMEIDA, E. S. DE; CASTRO, C. G. J. DE; VIEIRA, C. A. L. Distritos Sanitários : Concepção e OrganizaçãoSão Paulo, 1988.

AMAZONAS, S. DO E. DE S. Indicadores de Saúde - Aspectos Conceituais, 2012.

BLUMBERG, M. S. Risk Adjusting Health Care Outcomes: A Methodologic Review. Medical Care Research and Review, v. 43, n. 2, p. 351-393, 1 jan. 1986.

CARVAlHO, I. et al. Estudo da Gravidade dos Casos pelo Índice de Comorbidade de Charlson corrigido para Idade (ICC-I) nos Hospitais da Região de Ribeirão Preto nos Anos de 2007 e 2008. 10 Congresso Brasileiro de Saúde Coletiva, 2012.

CARVAlHO, I. et al. Ferramentas Computacionais para Análise de Dados no Auxílio à Gestão Hospitalar na Região de Ribeirão Preto. XIII Workshop de Informática Médica, 2013a.

CARVALHO, I. et al. Predição de Risco de Óbito por meio do Índice de Comorbidade de Charlson segundo Tipo de Atendimento. Congresso Ibero-Americano de Epidemiologia e Saúde Pública, $2013 b$.

CHARLSON, M. et al. Validation of a combined comorbidity index. Journal of Clinical Epidemiology, v. 47, n. 11, p. 1245-1251, nov. 1994.

CHARLSON, M. E. et al. A new method of classifying prognostic comorbidity in longitudinal studies: Development and validation. Journal of Chronic Diseases, v. 40, n. 5, p. 373-383, jan. 1987.

CHU, Y.-T.; NG, Y.-Y.; WU, S.-C. Comparison of different comorbidity measures for use with administrative data in predicting short- and long-term mortality. BMC health services research, $v$. 10, p. 140, jan. 2010.

CQH. Programa de Avaliação e Controle da Qualidade do Atendimento Médico-Hospitalar. [s.l: s.n.].

CQH. Manual de Indicadores de Enfermagem - NAGEH. São Paulo: [s.n.]. Disponível em: <http://www.cqh.org.br/>.

CSS. CSS, 2013. Disponível em: <http://www.w3.org/Style/CSS/>

DE GROOT, V. et al. How to measure comorbidity. a critical review of available methods. Journal of clinical epidemiology, v. 56, n. 3, p. 221-9, mar. 2003.

FARLEY, J. F.; HARLEY, C. R.; DEVINE, J. W. A comparison of comorbidity measurements to predict healthcare expenditures. The American journal of managed care, v. 12, n. 2, p. 110-9, fev. 2006.

FELDMAN, L. B.; GATTO, M. A. F.; CUNHA, I. C. K. O. História da evolução da qualidade hospitalar: dos padrões a acreditação. Acta Paulista de Enfermagem, v. 18, n. 2, p. 213-219, jun. 2005.

GONÇALVES, E. L. Gestão hospitalar: administrando o hospital moderno. São Paulo: Saraiva, 2006. p. 135 
HITOMI, W. Y. et al. O Índice de Comorbidade de Charlson como ferramenta do Observatório Regional de Atenção Hospitalar. XIII Congresso Brasileiro de Informática em Saúde, p. 19-23, 2012.

HTML. HTML, 2013. Disponível em: 〈http://www.w3.org/html/>

IUCIF JR, N.; ROCHA, J. S. Y. Estudo da desigualdade na mortalidade hospitalar pelo índice de comorbidade de Charlson. Revista de Saúde Pública, v. 38, n. 6, p. 780-786, dez. 2004.

LAURENTI, R. Análise da informação em saúde: 1893-1993, cem anos da Classificação Internacional de Doenças. Revista de Saúde Pública, v. 25, n. 6, dez. 1991.

MARTINS, M. Uso de medidas de comorbidades para predição de risco de óbito em pacientes brasileiros hospitalizados. Revista de Saúde Pública, v. 44, n. 3, p. 448-456, jun. 2010.

MARTINS, M.; BLAIS, R.; LEITE, I. DA C. Mortalidade hospitalar e tempo de permanência: comparação entre hospitais públicos e privados na região de Ribeirão Preto, São Paulo, Brasil. Cadernos de Saúde Pública, v. 20, p. S268-S282, 2004.

MARTINS, M.; BLAIS, R.; MIRANDA, N. N. DE. Avaliação do índice de comorbidade de Charlson em internações da região de Ribeirão Preto, São Paulo, Brasil. Cadernos de Saúde Pública, v. 24, n. 3, p. 643-652, mar. 2008.

MARTINS, M.; TRAVASSOS, C.; NORONHA, J. C. DE. Sistema de Informações Hospitalares como ajuste de risco em índices de desempenho. Revista de Saúde Pública, v. 35, n. 2, p. 185-192, abr. 2001.

MATHIAS, T. A. D. F.; SOBOLL, M. L. DE M. S. Confiabilidade de diagnósticos nos formulários de autorização de internação hospitalar. Revista de Saúde Pública, v. 32, n. 6, p. 526-532, dez. 1998.

MENDES, E. V. As redes de atenção à saúde. [s.l: s.n.]. v. 15p. 2297-2305

MINISTÉRIO DA SAÚDE. História e Evolução dos Hospitais. Rio de Janeiro: [s.n.].

MINISTÉRIO DA SAÚDE. Conceitos e Definições em SaúdeBrasília, 1977.

MINISTÉRIO DA SAÚDE. Lei $\mathbf{n}^{0} \mathbf{8 . 0 8 0}$, de 19 de setembro de 1990. Dispõe sobre as condições para a promoção, proteção e recuperação da saúde, a organização e o funcionamento dos serviços correspondentes e dá outras providências., 1990.

MINISTÉRIO DA SAÚDE. Sistema Único de Saúde (SUS) : princípios e conquistas, 2000.

MINISTÉRIO DA SAÚDE. Portaria $\mathbf{N}^{\mathbf{0}}$ 2224/GM, de 5 de dezembro de 2002. Estabelece o sistema de classificação hospitalar do Sistema Único de Saúde, 2002a. Disponível em: <http://dtr2001.saude.gov.br/sas/PORTARIAS/Port2002/Gm/GM-2224.htm>

MINISTÉRIO DA SAÚDE. Portaria nº 312, de 30 de abril de 2002, 2002b.

ORACLE. $\quad 2013 . \quad$ Java, Disponível em: <http://www.oracle.com/br/technologies/java/overview/index.html>

PEREIRA, S. D. Conceitos e Definições em Epidemiologia importantes para Vigilância Sanitária, 2007. 
PHPণ. Manual do PHP, 2013. Disponível em: <http://php.net/manual/pt_BR/index.php>

QUAIL, J. M. et al. Comparing comorbidity measures for predicting mortality and hospitalization in three population-based cohorts. BMC health services research, v. 11, n. 1, p. 146, jan. 2011.

QUAN, H. et al. Coding Algorithms for Defining Comorbidities in ICD-9-CM and ICD-10 Administrative Data. Medical Care, v. 43, n. 11, p. 1130-1139, nov. 2005.

ROCHA, J. S. Y. Utilização de leitos hospitalares gerais em Ribeirão Preto, São Paulo (Brasil). Revista de Saúde Pública, v. 9, n. 4, dez. 1975.

SANTOS, I. S.; UGÁ, M. A. D.; PORTO, S. M. O mix público-privado no Sistema de Saúde Brasileiro: financiamento, oferta e utilização de serviços de saúde. Ciência \& Saúde Coletiva, v. 13, n. 5, p. 1431-1440, out. 2008.

SIMÖES, B. et al. Controle de qualidade de dados hospitalares, Ribeiräo Preto, SP. Medicina (Ribeiräo Preto) 24.1, p. 26-32, 1991.

THOMAS, J. W. Risk Adjustment for Measuring Health Care Outcomes, 3rd edition. International Journal for Quality in Health Care, v. 16, n. 2, p. 181-182, 1 abr. 2004.

TRAVASSOS, C.; NORONHA, J. C. DE; MARTINS, M. Mortalidade hospitalar como indicador de qualidade: uma revisão. Ciência \& Saúde Coletiva, v. 4, n. 2, p. 367-381, 1999.

TREVISO, P.; BRANDÃO, F. H.; SAITOVITCH, D. Construção de indicadores em serviços de saúde. Revista Administração Saúde, v. 11, p. 182-186, 2009.

UNICAMP; MINISTÉRIO DA SAÚDE. Aglomerados de Saúde: São Paulo, 2002.

VERAS, C. M. T.; MARTINS, M. S. A confiabilidade dos dados nos formulários de Autorização de Internação Hospitalar (AIH), Rio de Janeiro, Brasil. Cadernos de Saúde Pública, v. 10, n. 3, p. 339 355, set. 1994.

VINCI, A. L. T. Análise e Avaliação do Controle de Qualidade de Dados Hospitalares na Região de Ribeirão Preto. [s.l: s.n.].

YAZLLE ROCHA, J. S.; SIMÕES, B. J. G.; GUEDES, G. L. M. Assistência hospitalar como indicador da desigualdade social. Revista de Saúde Pública, v. 31, n. 5, p. 479-487, out. 1997. 


\section{ANEXO A}

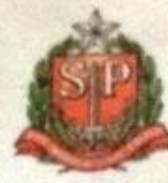

HOSPITAL DAS CLINICAS DA FACULDADE DE MEDICINA DE RIBEIRÃO PRETO DA UNIVERSIDADE DE SÄO PAULO

Oficio $\mathrm{n}^{\circ} 1868 / 2012$

CEP/MGV

PROCESSO HCRP n $5056 / 2012$

Prezados Pesquisadores,

ATRAVÉS DA ANÁLISE DE COMORBIDADES PARA CLASSIIICAÇ̄o DOS SERVICOS

$$
\text { O trabalho intitulado "AJUSTE DO CÁLCULO DE RISCO }
$$
DE UMA REDE DE ASSISTENCIA HOSPITALAR", foi analisado pelo Comitê de Ética em Pesquisa, em sua 346" Reuniầo Ordinária realizada em 21/05/2012, e enquadrado na categoria: APROVADO, bem como a dispensa do Termo de Consentimento Livre e Esclarecido.

Este Comite segue integralmente a Conferencia Internacional de Harmonizaçao de Boas Práticas Clinicas (IGH-GCP), bem como a Resoluçao $n^{\circ}$ 196/96 CNS/MS.

Lembramos que devem ser apresentados a este CEP, o Relatório Parcial e o Relatório Final da pesquisa. De acordo com Carta Circular $n^{\circ}$ 003/2011/CONEP/CNS, datada de 21/03/2011, o sujeito de pesquisa ou seu representante, quando for o caso, devera rubricar todas as folhas do Termo de Consentimento Livre e Esclarecido - TCLE - apondo sua assinatura na última do referido Termo; o pesquisador responsavel devera da mesma forma, rubricar todas as folhas do Termo de Consentimento Livre e Esclarecido - TCLE - apondo sua assinatura na ultima página do referido Termo.

Atenciosamente.

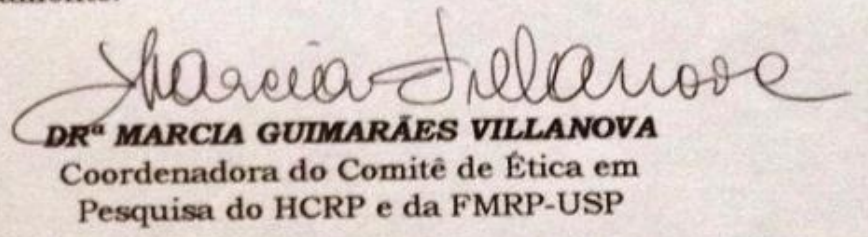

Ilustrissimos Senhores

ISABELLE CARVALHO

PROF. DR. JUAN STUARDO YAZLLE ROCHA (Orientador)

Depto. de Medicina Social 